Publ. RIMS, Kyoto Uniz.

15 (1979), 401-151

\title{
Asymptotic Behavior and Stability of Solutions of Semilinear Diffusion Equations
}

By

\author{
Hiroshi MATANO*
}

\section{$\S \mathbb{1}$. Introduction}

This paper is divided into two chapters. In the first chapter ( $\S 2$ $\sim \S 4)$ we study the asymptotic behavior of solutions of semilinear diffusion equations - in particular their behavior around unstable equilibrium solutions, so as to get a clearer understanding of the situation where those unstable equilibrium solutions are placed. The results will then be applied to making some stability criteria as well as to establishing theorems on the existence of stable equilibrium solutions and on the structure of multiple equilibrium solutions. In the second chapter ( $\$ 5$, $\S 6)$ we are confined to Neumann problems and discuss the possibility of the existence of non-constant stable equilibrium solutions.

Let $D$ denote a bounded domain in $\boldsymbol{R}^{n}$ with a smooth boundary $\partial D$. What we shall deal with first are initial-boundary value problems of the type

(1.1a) $\frac{\partial u}{\partial t}=L u+f(x, u)$ in $D \times(0, T)$,

(1. 1b) $\quad u(x, 0)=u_{0}(x)$ on $D$,

(1. 1c) $\quad \alpha(x) u+\{1-\alpha(x)\} \frac{\partial u}{\partial \nu}=\beta(x)$ nn $\partial D \times\left(0, T^{\prime}\right)$,

where $L$ is a second order uniformly elliptic operator of the form

$$
L u=\sum_{i, j=1}^{n} \frac{\partial}{\partial x_{i}}\left\{a_{i j}(x) \frac{\partial u}{\partial x_{j}}\right\}
$$

and $\partial / \partial \nu$ denotes the outward conormal derjvative. It is alivays assumed

Communicated by S. Matsuura, April 12, 1977.

* Research Institute for Mathematical Sciences, Kyoto University.

Present address: Department of Mathematics, University of Tokyo. 
that $0 \leqq \alpha \leqq 1$ everywhere on $\partial D$. A function $v=v(x)$ is said to be an equilibrium solution, or a steady-state solution, of (1.1) if it satisfies the following elliptic boundary value problem:

$$
\begin{aligned}
& L v+f(x, v)=0 \quad \text { in } D, \\
& \alpha v+(1-\alpha) \frac{\partial v}{\partial \nu}=\beta \text { on } \partial D .
\end{aligned}
$$

Throughout this paper only real-valued classical solutions are considered.

Our main result in Chap. I is related to the question of how a solution of (1.1) will behave in the long run when starting from nearby an unstable equilibrium solution. In short, we shall show that above an unstable solution $v$ of (1.2) there exists a minimal solution $v^{+}$in a certain sense and that for any continuous function $\psi$ satisfying $v<\psi \leqq v^{+}$ the solution of (1.1) with initial data $u_{0}=\psi$ converges to $v^{+}$as $t$ increases. Here, roughly speaking, $v^{+}$must often be replaced by $+\infty$ according to circumstances. A similar assertion holds for a maximal solution below $v$.

The main result in Chapter II is on the stability of solutions of the following Neumann problem:

$$
\begin{aligned}
& \Delta v+f(v)=0 \text { in } D, \\
& \frac{\partial v}{\partial n}=0 \text { on } \partial D,
\end{aligned}
$$

where $\partial / \partial n$ denotes the outer normal derivative. It shall be shown that if $D$ is a convex domain or one of other certain kinds of domains then any non-constant solution of (1.3) is, if it exists, unstable.

As regards the stability of equilibrium solutions, D. H. Sattinger has shown in [12] how stability and instability are characterized in terms of upper and lower solutions. The characterization of stability in this direction presents us a clear understanding of the global behavior of solutions around stable and unstable equilibrium solutions. But so far, it seems that stability-including instability-of solutions of (1.2) has not yet been completely characterized by means of those upper and lower solutions, or supersolutions and subsolutions in [3]. In this present paper, taking advantage of the self-adjointness of the elliptic 
operator $L$, we shall make quite another approach to the understanding of the global behavior of solutions which will provide us with a complete knowledge of "the extent of instability". The fundamental technique for the discussion of the asymptotic behavior of solutions is given in the next section. One of our contributions here is to reveal the extent of instability in pretty general situations, and another is to emphasize the importance of this result.

In $\S 2$, we prove, as is mentioned just above, some fundamental theorems on the asymptotic behavior of solutions which are based upon estimates of fundamental solutions, Schauder's estimates, and the so-called energy methods. For convenience' sake, we employ the idea of $\omega$-limiting sets in the discussion. What is important is the fact that the $\omega$-limiting set of a solution of (1.1) consists only of the solutions of (1.2) and that in many cases it is not empty (Theorem 2.8). We usually discuss the behavior of solutions simply in the topology of $C^{1}(\bar{D}) \cap C^{2}(D)$ and never in a stronger one even if possible, since it is sufficient for the essential part of the following argument.

In $\S 3$, the main result in this chapter is presented (Theorem 3.1). It should be noted that no further assumption than a smoothness condition is made on the nonlinear term $f$. Applications of this theorem are found mainly in $\S 4$, but also the results in $\S 6$ are essentially (if not directly) based upon this theorem.

In $\S 4$, theorems on the existence and on the structure of solutions of (1. 2) are given. We first prove that a subset of $C^{1}(\bar{D}) \cap C^{2}(D)$ which is stable as a set in a certain sense contains at least one stable solution (Theorem 4.2). Next we show that between two unstable solutions below and above there exists at least one stable solution (Theorem 4.3) and that between two stable solutions exists at least one solution (Theorem 4.4). It is H. Amann who has proved the existence of minimal and maximal solutions between a lower solution below and an upper solution above (see [1]). Our theorems in $\S 3, \S 4$ are partly analogous to [1] as well as to [12], but include stronger results that probably can hardly be obtained by their methods, namely the monotone iteration methods. From a practical point of view we must note Corollary 4.5 and Remark 4.6, which are sometimes useful for investigating the stability of 
solutions. For example, given a maximal solution $v$ of (1.2) (i.e., a solution above which there lies no other solution), one can see, from Remark 4. 6 , that it is quite sufficient to conclude the instability of $v$ if he only finds a continuous function $\psi$ which makes the solution of (1.1) with initial data $\psi$ blow up to $+\infty$ in a finite time or diverge to $+\infty$ as $t \rightarrow \infty$ (here we assume $n=1$ and that $f$ is independent of $x$ ).

In $\S 5$, we consider (1.3), which is a special case of (1.2), and present one of the main theorems in Chap. II (Theorem 5.1). This theorem is a kind of generalization of N. Chafee's result [4; Section 6] on the instability of solutions of (1.3). He has proved that $f$ being of class $C^{2}$ any isolated non-constant solution of (1.3) is unstable provided that $n=1$. Our theorem here, which contains the same kind of result for convex domains in $\boldsymbol{R}^{n}$, of course includes his result and, moreover, is stronger than his even if restricted to one-dimensional cases, for it is all the same applicable to non-isolated solutions. One also finds other two types of domains in which any non-constant solution is unstable, namely a body of rotation with a convex section and a domain bounded by two concentric spheres. ( $\partial D$ is always assumed to be smooth.)

In $\S 6$, it is shown that when $n \geqq 2$ there are actually cases where (1.3) has a non-constant stable solution (Theorem 6.2). We give sufficient conditions on the domain $D$ and the function $f$ for the existence of such a solution. This result is obtained by applying Theorem 4.2 to a certain invariant subset of $C^{1}(\bar{D}) \cap C^{2}(D)$ which contains no constant function.

The author wishes to express his gratitude to Professor Masaya Yamaguti for continued encouragement and guidance throughout the course of this work and to Professor Masayasu Mimura for stimulating suggestions by which the development of Chapter II was motived. He also thanks Professor Seizô Itô for a useful remark on the estimates of fundamental solutions. 


\section{Chapter I. General Theory}

\section{$\S$ 2. Preliminaries}

Our assumptions concerning the equations (1.1) and (1.2) are all put together as follows:

(A) 1) $D$ is bounded and its boundary is sufficiently smooth, say of class $C^{3}$.

2) Each $a_{i j}(x)$ is smooth, say all its second derivatives are uniformly Hölder continuous in $\bar{D}$ (of exponent $\gamma, 0<\gamma<1$ ), and the matrix $\left(a_{i j}\right)$ is uniformly positive definite and symmetric.

$3)$ Both $\alpha(x)$ and $\beta(x)$ belong to $C^{2}(\partial D)$, and $0 \leqq \alpha \leqq 1$ everywhere on $\partial D$.

4) $f(x, u)$ is of class $C^{2}$ in $u$. Moreover, $f$ and $\partial f / \partial u$ are Hölder continuous (of exponent $\gamma$ ) in $\bar{D} \times R$.

We make no further assumption throughout this section, while some of the results in next two sections require slightly stronger boundary conditions. Smoothness conditions in (A) may be relaxed somewhat, for which we shall not care in this paper.

For the purpose of convenience in making a priori estimates and in discussing the asymptotic behavior of solutions, let us introduce some function spaces.

First, for description of the asymptotic behavior: Given an interval $I$ in $\boldsymbol{R}^{1}$ and a metric space $X$, we denote by $C(I ; X)$ the space of all $X$-valued continuous functions on $I$ with the topology of uniform convergence on compact subintervals.

Secondly, for a priori estimates: Given a closed interval $I$, a compact set $K$ in $\bar{D}$ and a positive number $\gamma<1$, we introduce the notation

$$
|g|_{r, I, K}=\sup _{\substack{x \in K \\ t \in I}}|g(x, t)|+\sup _{\substack{x, x^{\prime} \in K \\ t, t^{\prime} \in I}} \frac{\left|g(x, t)-g\left(x^{\prime}, t^{\prime}\right)\right|}{\left(\left|x-x^{\prime}\right|^{2}+\left|t-t^{\prime}\right|\right)^{r / 2}},
$$

where $g$ is a function defined on $K \times I$. We also need the notation 


$$
|g|_{2+r, I, K}=\sup _{\substack{x \in K \\ t \in I}}|g|+\sum_{i, j=1}^{n}\left|\frac{\partial^{2} g}{\partial x_{i} \partial x_{j}}\right|_{r, I, K}+\left|\frac{\partial g}{\partial t}\right|_{\gamma, I, K} .
$$

The space of all functions with finite norm $|g|_{r, I, \bar{D}}$ is denoted by $C^{r}(\bar{D} \times I)$. And $C^{2+r}\left(D \times\left(t_{1}, t_{2}\right]\right)$ denotes the Fréchet space defined by the system of seminorms $|g|_{2+r,\left[s, t_{2}\right], K}$ where $K$ varies over all the compact sets in $D$ and $s$ over $\left(t_{1}, t_{2}\right]$. It is not difficult to see that $C^{1}(\bar{D} \times I)$ is embedded continuously both into $C^{r}(\bar{D} \times I)$ and into $C\left(I ; C^{1}(\bar{D})\right)$, and that $C^{2+r}\left(D \times\left(t_{1}, t_{2}\right]\right)$ completely continuously into $C\left(\left(t_{1}, t_{2}\right] ; C^{2}(D)\right)$.

We are now ready to formulate our a priori estimates-a pretty weakened form of what is known as the interior estimates of the Schauder type:

Lemma 2. 1. Let $g(x, t)$ belong to $C^{r}\left(\bar{D} \times\left[t_{1}, t_{2}\right]\right)$ and let $u(x, t)$ be a continuous function on $\bar{D} \times\left[t_{1}, t_{2}\right]$ satisfying.

$$
\frac{\partial u}{\partial t}=L u+g(x, t) \quad \text { in } \quad D \times\left(t_{1}, t_{2}\right]
$$

Then $u$ belongs to $C^{2+r}\left(D \times\left(t_{1}, t_{2}\right]\right)$. Moreover, given any compact set $K \subset D$ and any $s \in\left(t_{1}, t_{2}\right]$, there exists a constant $C>0$ such that

$$
|u|_{2+r,\left[s, t_{2}\right], K} \leqq C\left(\|u\|_{L^{\infty}\left(D \times\left(t_{1}, t_{2}\right)\right)}+|g|_{r,\left[t_{1}, t_{2}\right], \bar{D}}\right)
$$

for any continuous function $u$ satisfying the above equation.

Our assumption on $D$ and $L$ is quite enough for the estimates of this type. See A. Friedman [5; Theorems 5 and 9 in Chapter 3] for details.

Let $U=U(x, y, t)$, defined on $\bar{D} \times \bar{D} \times(0, \infty)$, be the fundamental solution of the equation

$$
\frac{\partial u}{\partial t}=L u \quad \text { in } \quad D \times(0, \infty)
$$

with the boundary condition

$$
\alpha u+(1-\alpha) \frac{\partial u}{\partial \nu}=0 \quad \text { on } \quad \partial D \times(0, \infty),
$$

where $L$ and $\alpha$ are as in (A). We owe the following lemma to S. Itô [private communication]. (This result can naturally be obtained in the 
course of constructing the fundamental solution in a bounded domain; see $[8 ; \S 3, \S 4]$ for the construction of fundamental solutions.)

Lemma 2. 2. $U, \nabla_{x} U$ and $\partial U / \partial t$ are continuous in $\bar{D} \times \bar{D} \times(0, \infty)$, and $U(x, y, t)=U(y, x, t)>0$ on $D \times D \times(0, \infty)$, where $\nabla_{x}=\left(\partial / \partial x_{1}\right.$, $\left.\partial / \partial x_{2}, \cdots, \partial / \partial x_{n}\right)$. Moreover, given any $t_{0}>0$, there exist positive constants $M_{i}(i=1,2,3)$ such that

$$
\begin{aligned}
& \sup _{x \in \bar{D}} \int_{D} U(x, y, t) d y \leqq M_{1}, \\
& \sup _{x \in \bar{D}} \int_{D}\left|\frac{\partial U(x, y, t)}{\partial t}\right| d y \leqq M_{2} t^{-1},
\end{aligned}
$$

for $0<t \leqq t_{0}$ and that

$$
\sup _{x \in \bar{D}} \int_{0}^{t_{0}} d \tau \int_{D}\left|\nabla_{x} U(x, y, \tau)\right| d y \equiv M_{3}<\infty
$$

We introduce linear operators $T$ and $S$ with parameter $t$ : For each $t \geqq 0, T(t)$ is an operator on $L^{\infty}(D)$ defined by

$$
[T(t) w](x)=\int_{D} U(x, y, t) w(y) d y,
$$

where we understand $T(0) w=w . S(t)$ is defined by

$$
[S(t) g](x)=\int_{0}^{t} d \tau \int_{D} U(x, y, t-\tau) g(y, \tau) d y,
$$

and here we understand $S(0) g=0$. These integrals are easily seen to induce such operators as

(2. 4a) $T: L^{\infty}(D) \rightarrow C^{r}\left(\bar{D} \times\left[\delta, t_{0}\right]\right) \cap C\left(\left[\delta, t_{0}\right] ; C^{1}(\bar{D})\right)$

(2. 4b) $S: L^{\infty}\left(D \times\left(0, t_{0}\right)\right) \rightarrow C^{\gamma}\left(\bar{D} \times\left[0, t_{0}\right]\right) \cap C\left(\left[0, t_{0}\right] ; C^{1}(\bar{D})\right)$ for any given $0<\delta<t_{0}$ and $0<\gamma<1$. Moreover;

Lemma 2. 3. The operators in (2.4) are both compact.

Proof. By virtue of the continuity of $\partial U / \partial t$ and $\nabla_{x} U$ on the compact set $\bar{D} \times \bar{D} \times\left[\delta, t_{0}\right], T$ is compact if regarded as a mapping from $L^{\infty}(D)$ into $C^{1}\left(\bar{D} \times\left[\delta, t_{0}\right]\right)$. Since $C^{1}\left(\bar{D} \times\left[\delta, t_{0}\right]\right)$ is embedded 
continuously into $C^{r}\left(\bar{D} \times\left[\delta, t_{0}\right]\right) \cap C\left(\left[\delta, t_{0}\right] ; C^{1}(\bar{D})\right)$, the compactness of the mapping in (2.4a) follows.

Well, let

$$
A=\left\{g \in L^{\infty}\left(D \times\left(0, t_{0}\right)\right) ;\|g\|_{\infty} \leqq 1\right\} .
$$

Using (2.1) and (2.3) we get

$$
\sup _{\substack{x \in \bar{D} \\ t \in\left[0, t_{0}\right]}}\left\{|[S(t) g](x)|+\left|\nabla_{x}[S(t) g](x)\right|\right\} \leqq M_{1} t_{0}+M_{3},
$$

for all $g \in A$. As $\bar{D}$ is compact and as $\nabla_{x} U$ is continuous on $\bar{D} \times\left(0, t_{0}\right]$, it also follows from (2.3) that given any $\varepsilon>0$ there exists a $d>0$ such that

$$
\left|\nabla_{x}[S(t) g](x)-\nabla_{x}[S(t) g]\left(x^{\prime}\right)\right|<\varepsilon
$$

for all $g \in A, t \in\left[0, t_{0}\right]$ and $x, x^{\prime} \in \bar{D}$ satisfying $\left|x-x^{\prime}\right|<d$. (Note that $\left|\nabla_{x} S(t) g\right|$ itself is sufficiently small if $t$ is sufficiently small.) On the other hand, for any $0 \leqq t_{1}<t_{2} \leqq t_{0}$ and any $g \in A$

$$
\begin{aligned}
& \left|\left[S\left(t_{2}\right) g\right](x)-\left[S\left(t_{1}\right) g\right](x)\right| \\
& =\mid \int_{0}^{t_{2}} d \tau \int_{D} U\left(x, y, t_{2}-\tau\right) g(y, \tau) d y \\
& -\int_{0}^{t_{1}} d \tau \int_{D} U\left(x, y, t_{1}-\tau\right) g(y, \tau) d y \\
& \leqq \int_{\max \left(t_{1}-\Delta t, 0\right)}^{t_{2}} d \tau \int_{D}\left|U\left(x, y, t_{2}-\tau\right) g(y, \tau)\right| d y \\
& +\int_{\max \left(t_{1}-\Delta t, 0\right)}^{t_{1}} d \tau \int_{D}\left|U\left(x, y, t_{1}-\tau\right) g(y, \tau)\right| d y \\
& +\int_{0}^{\max \left(t_{1}-\Delta t, 0\right)} d \tau \int_{D}\left|U\left(x, y, t_{2}-\tau\right)-U\left(x, y, t_{1}-\tau\right)\right||g(y, \tau)| d y \\
& \leqq 3 M_{1} \Delta t+\int_{\min \left(t_{1}, \Delta t\right)}^{t_{1}} d \tau \int_{D}|U(x, y, \tau+\Delta t)-U(x, y, \tau)| d y \\
& \leqq 3 M_{1} \Delta t+\int_{\min \left(t_{1}, \Delta t\right)}^{t_{1}} d \tau \int_{D} d y \int_{0}^{\Delta t}\left|\frac{\partial U(x, y, \tau+s)}{\partial t}\right| d s,
\end{aligned}
$$

where $\Delta t=t_{2}-t_{1}$. Using (2.2), we get

$$
\begin{aligned}
\int_{D} d y \int_{0}^{\Delta t}\left|\frac{\partial U(x, y, \tau+s)}{\partial t}\right| d s & =\int_{0}^{\Delta t} d s \int_{D}\left|\frac{\partial U(x, y, \tau+s)}{\partial t}\right| d y \\
& \leqq M_{2} \int_{0}^{\Delta t} \frac{d s}{\tau+s}<M_{2} \Delta t \tau^{-1}
\end{aligned}
$$


Hence, an elementary calculation yields

$$
\begin{aligned}
\sup _{x \in \bar{D}} \mid & {\left[S\left(t_{2}\right) g\right](x)-\left[S\left(t_{1}\right) g\right](x) \mid } \\
& \leqq\left(3 M_{1}+M_{2} \log \frac{t_{1}}{\min \left(t_{1}, \Delta t\right)}\right) \Delta t \\
& \leqq\left\{3 M_{1}+M_{2}\left(\left|\log t_{0}\right|+\left|\log \left(t_{2}-t_{1}\right)\right|\right)\right\}\left(t_{2}-t_{1}\right)
\end{aligned}
$$

for all $g \in A$ and $0 \leqq t_{1}<t_{2} \leqq t_{0}$. Seeing (2.5) and (2.7), we easily find that $\{S g\}_{g \in A}$ is relatively compact in $C^{r}\left(\bar{D} \times\left[0, t_{0}\right]\right)$ for any $0<\gamma<1$. It remains to show the relative compactness of $\{S g\}_{g \in A}$ in $C\left(\left[0, t_{0}\right]\right.$; $\left.C^{1}(\bar{D})\right)$. For each fixed $t \in\left[0, t_{0}\right], S(t) g$ is a function of $x \in \bar{D}$, and it remains in a compact set of $C^{1}(\bar{D})$ as $t$ varies in $\left[0, t_{0}\right]$ and $g$ in $A$, by virtue of (2.5), (2.6) and Ascoli-Arzelà's theorem. So what we are to prove is the equicontinuity of $\{S g\}_{g \in A}$ in $t$ with respect to the topology of $C^{1}(\bar{D})$. Since the norm of $C^{1}(\bar{D})$ and that of $C(\bar{D})$ are equivalent on the set $\left\{S(t) g \mid 0 \leqq t \leqq t_{0}, g \in A\right\}$ as it is relatively compact in $C^{1}(\bar{D})$, we have only to prove the equicontinuity in the topology of $C(\bar{D})$, which follows immediately from (2.7). Thus the relative compactness of $\{S g\}_{g \in A}$ in $C^{r}\left(\bar{D} \times\left[0, t_{0}\right]\right) \cap C\left(\left[0, t_{0}\right] ; C^{1}(\bar{D})\right)$ is established. Hence the compactness of the operator $S$ in $(2.4 \mathrm{~b})$.

Q.E.D.

Now we turn to the initial-boundary value problem (1.1). Converting (1.1) to an integral equation and then using the successive approximation method, one easily finds that for any bounded continuous initial data the unique existence of the solution of (1.1) is guaranteed so long as $t$ remains small. Therefore, for each $t \geqq 0$, an operator $Q(t)$ on $\mathscr{B}^{0}(D)$ $\equiv L^{\infty}(D) \cap C(D)$ can be defined as follows:

$$
\left[Q(t) r^{\prime}\right](x)=u_{w}(x, t) \text { for } \quad w \in \mathscr{B}^{0}(D),
$$

where $u_{w}$ is the solution of (1.1) with initial data $u_{0}=w$. For each fixed $t^{\prime} \geqq 0, Q\left(t^{\prime}\right)$ is generally a nonlinear operator and its domain - denoted by Dom $\left(Q\left(t^{\prime}\right)\right)$ - is not necessarily the whole space $\mathscr{B}^{0}(D)$. A function $w$ belongs to $\operatorname{Dom}\left(Q\left(t^{\prime}\right)\right)$ if and only if $u_{w}$ can be extended farther than $t=t^{\prime}$ as a solution of (1.1); in other words, if and only if $u_{w}$ does not blow up in the time interval $\left[0, t^{\prime}\right]$. So we have $\operatorname{Dom}(Q(0))=\mathscr{B}^{0}(D)$, and one can easily see 


$$
\begin{aligned}
& \operatorname{Dom}\left(Q\left(t_{1}\right)\right) \supset \operatorname{Dom}\left(Q\left(t_{2}\right)\right), \\
& Q\left(t_{2}\right)=Q\left(t_{2}-t_{1}\right) Q\left(t_{1}\right),
\end{aligned}
$$

for any $0 \leqq t_{1} \leqq t_{2}$.

Theorem 2.4. Let (A) hold and let $B$ be a bounded set in $\mathscr{B}^{0}(D)$. Then

i) there exists a positive number $t_{0}$ such that $B \subset \operatorname{Dom}\left(Q\left(t_{0}\right)\right)$ and that $\{Q w\}_{w \in B}$ is bounded in $L^{\infty}\left(D \times\left(0, t_{0}\right)\right)$, where Qw denotes a function of $x, t$ expressed by $[Q(t) w](x)$;

ii) if $\{Q w\}_{w \in B}$ is bounded in $L^{\infty}\left(D \times\left(0, t_{1}\right)\right)$ for some $t_{1}>0$, it is relatively compact in $C\left(\left(0, t_{1}\right] ; C^{1}(\bar{D}) \cap C^{2}(D)\right)$.

We prove this theorem only in the case where $\beta \equiv 0$, for otherwise (1.1) can be reduced to this case by subtracting from $u$ a function $w_{0} \in C^{1}(\bar{D}) \cap C^{2}(D)$ which satisfies the same boundary condition as (1. 2b) and makes $L w_{0}$ uniformly Hölder continuous in $\bar{D}$. (We can indeed find such a $w_{0}$, since given any uniformly Hölder continuous data $\varphi$ the elliptic equation $L w_{0}=\varphi$ with the boundary condition (1.2b) is solvable except that when $\alpha \equiv 0, \varphi$ must be so chosen as to satisfy

$$
\int_{D} \varphi d x=\int_{\partial D} a(\tilde{x}) \beta(\tilde{x}) d \tilde{x},
$$

where

$$
a(\widetilde{x})=\left[\sum_{i}\left\{\sum_{j} a_{i j}(\widetilde{x}) n_{j}(\widetilde{x})\right\}^{2}\right]^{1 / 2}
$$

and here $\left(n_{j}(\tilde{x})\right)$ denotes the outer normal to $\partial D$ at $\widetilde{x}$.) Note that if the assumption (A) holds for (1.1) it is still the same with the reduced problem

$$
\left\{\begin{array}{l}
\frac{\partial \bar{u}}{\partial t}=L \bar{u}+\bar{f}(x, \bar{u}) \\
\bar{u}(x, 0)=\bar{u}_{0}(x) \\
\alpha \bar{u}+(1-\alpha) \frac{\partial \bar{u}}{\partial \nu}=0
\end{array}\right.
$$

where 


$$
\bar{f}(x, \bar{u})=f\left(x, \bar{u}+w_{0}(x)\right)+L w_{0}(x) .
$$

For $L w_{0}$ is uniformly Hölder continuous.

Proof of Theorem 2.4. As is mentioned above, we can assume $\beta \equiv 0$ without any loss of generality.

i) Let $f_{1}(u), f_{2}(u)$ be locally Lipschitz continuous functions satisfying

$$
\begin{aligned}
& f_{1}(u) \leqq \min _{x \in \bar{D}}(0, f(x, u)), \\
& f_{2}(u) \geqq \max _{x \in \bar{D}}(0, f(x, u)),
\end{aligned}
$$

for all $u \in R$, and let us then consider the following ordinary differential equations:

$$
\begin{aligned}
& \left\{\begin{array}{l}
\frac{d u_{1}}{d t}=f_{1}\left(u_{1}\right), \quad t \geqq 0 \\
u_{1}(0)=-M,
\end{array}\right. \\
& \left\{\begin{array}{l}
\frac{d u_{2}}{d t}=f_{2}\left(u_{2}\right), \quad t \geqq 0 \\
u_{2}(0)=M,
\end{array}\right.
\end{aligned}
$$

where $M=\sup _{w \in B}\|w\|_{L^{\infty}(D)}$. Either of these equations has a solution at least on some interval $0 \leqq t \leqq t_{0}\left(t_{0}>0\right)$. Seeing (2.10), we have

$$
u_{1}(t) \leqq 0 \leqq u_{2}(t)
$$

for $0 \leqq t \leqq t_{0}$. Besides, regarded as functions of $x, t$, these solutions obviously satisfy $L u_{i}=0$ and $\partial u_{i} / \partial \nu=0 \quad(i=1,2)$. Considering these facts and using (2.10) again, we get, instead of (2.11) and (2.12), the following partial differential inequalities:

$$
\left\{\begin{array}{l}
\frac{\partial u_{1}}{\partial t} \leqq L u_{1}+f\left(x, u_{1}\right) \quad \text { in } D \times\left(0, t_{0}\right] \\
u_{1}(x, 0) \leqq w(x) \quad \text { on } \quad D \\
\alpha u_{1}+(1-\alpha) \frac{\partial u_{1}}{\partial \nu} \leqq 0 \text { on } \quad \partial D \times\left(0, t_{0}\right]
\end{array}\right.
$$




$$
\left\{\begin{array}{l}
\frac{\partial u_{2}}{\partial t} \geqq L u_{2}+f\left(x, u_{2}\right) \quad \text { in } \quad D \times\left(0, t_{0}\right] \\
u_{2}(x, 0) \geqq w(x) \quad \text { on } \quad D \\
\alpha u_{2}+(1-\alpha) \frac{\partial u_{2}}{\partial \nu} \geqq 0 \quad \text { on } \quad \partial D \times\left(0, t_{0}\right],
\end{array}\right.
$$

here $w$ is any element of $B$. Since $0 \leqq \alpha \leqq 1$, it follows from a standard comparison theorem that

$$
u_{1}(t) \leqq[Q(t) w](x) \leqq u_{2}(t)
$$

for all $x \in \bar{D}$ and $t \in\left(0, t_{0}\right]$. (See, for instance, M. H. Protter- H. F. Weinberger [11; Theorem 8 in Chapter 3], where the proof of the comparison theorem of this type is implicitly suggested.) Strictly speaking, this inequality should be understood to hold so long as $Q(t) w$ is definable, i.e., $w \in \operatorname{Dom}(Q(t))$, but as a matter of fact it is the case because any solution of (1.1) can be extended until it blows up or to $t=\infty$. Hence this completes the proof of i)

ii) Converting (1.1) into an integral equation in the usual manner, we get

$$
Q(t) w=T(t) w+S(t) \tilde{f}_{w},
$$

where

$$
\tilde{f}_{w}(x, t)=f(x,[Q(t) w](x)) .
$$

(Here the boundary integral term does not appear, for we have assumed that $\beta \equiv 0$.) As $\{Q w\}_{w \in B}$ is bounded in $L^{\infty}\left(D \times\left(0, t_{1}\right)\right)$, so is $\left\{\tilde{f}_{w}\right\}_{w \in B}$ because of the continuity of $f$. Therefore Lemma 2.3 can be applied to get the relative compactness of $\{Q w\}_{w \in B}$ in $C^{r}\left(\bar{D} \times\left[\delta, t_{1}\right]\right) \cap C\left(\left[\delta, t_{1}\right]\right.$; $\left.C^{1}(\bar{D})\right)$ for any given $\delta \in\left(0, t_{1}\right]$. Hence $\{Q w\}_{w \in B}$ is relatively compact in $C\left(\left[\delta, t_{1}\right] ; C^{1}(\bar{D})\right)$. On the other hand, as $f$ is uniformly Hölder continuous of exponent $\gamma$ in $x$ and Lipschitz continuous in $u,\left\{\tilde{f}_{w}\right\}_{w \in B}$ is bounded in $C^{r}\left(\bar{D} \times\left[\delta, t_{1}\right]\right)$. So it follows from Lemma 2.1 that $\{Q w\}_{w \in B}$ is bounded in $C^{2+r}\left(D \times\left(\delta, t_{1}\right]\right)$, hence relatively compact in $C\left(\left(\delta, t_{1}\right] ; C^{2}(D)\right)$. Combining these facts, and considering that $\delta$ is any number in $\left(0, t_{1}\right]$, we now obtain what is expected. Q.E.D. 
Corollary 2.5. Let (A) hold and let $\left\{v_{\lambda}\right\}_{\lambda \in A}$ be a set of solutions of (1.2). Suppose $\left\{v_{\lambda}\right\}_{\lambda \in A}$ is bounded in $\mathscr{B}^{0}(D)$. Then it is relatively compact in $C^{1}(\bar{D}) \cap C^{2}(D)$.

The proof is immediate, since $\left\{v_{\lambda}\right\}_{\lambda \in A}=\left\{Q(t) v_{\lambda}\right\}_{\lambda \in A}$ for $t>0$.

Lemma 2.6. Let (A) hold.

i) For each fixed $t>0$, the following mappings are continuous linear operators (cf. (2.4)):

$$
\begin{aligned}
& T(t): L^{2}(D) \rightarrow L^{2}(D), \\
& S(t): L^{\infty}\left(0, t ; L^{2}(D)\right) \rightarrow L^{2}(D) .
\end{aligned}
$$

ii) Let $B, t_{0}$ be as in Theorem 2. 4. Then there exists a constant $C>0$ such that for all $w_{1}, w_{2} \in B$,

$$
\sup _{0 \leqq t \leqq t_{0}}\left\|Q(t) w_{1}-Q(t) w_{2}\right\|_{L^{2}(D)} \leqq C\left\|w_{1}-w_{2}\right\|_{L^{2}(D)} .
$$

We omit the proof of this lemma, for such kinds of results are so popular. Suffice it to say that ii) is an immediate consequence of a usual successive approximation in the integral equation (2.13).

Our estimates obtained so far are not yet sufficient for the discussion of the asymptotic behavior of solutions; it is because they are merely local estimates (with respect to $t$ ). We need some more information on solutions which is in a sense global in $t$. For this purpose we introduce an energy form $J(u)$ associated with (1.1):

$$
\begin{aligned}
J(u)= & \int_{D}\left\{\frac{1}{2} \sum_{i, j=1}^{n} a_{i j} \frac{\partial u}{\partial x_{i}} \frac{\partial u}{\partial x_{j}}-F(x, u)\right\} d x \\
& +\int_{\partial D} a\left\{\frac{\alpha}{2} u^{2}+\frac{1-\alpha}{2}\left(\frac{\partial u}{\partial \nu}\right)^{2}-\beta u\right\} d \widetilde{x},
\end{aligned}
$$

where

$$
F(x, u)=\int_{0}^{u} f(x, u) d u
$$

and $a(\tilde{x})$ is as in (2.9). For any $t>0$ and any $w \in \operatorname{Dom}(Q(t))$ the 
form $J(Q(t) w)$ is definable.

Lemma 2. 7. Let (A) hold and let $t_{0}$ be any positive number. Then for any $w \in \operatorname{Dom}\left(Q\left(t_{0}\right)\right)$

$$
\frac{d}{d t} J(Q(t) w)=-\int_{D}\left\{\frac{\partial}{\partial t} Q(t) w\right\}^{2} d x, \quad 0<t \leqq t_{0} .
$$

Proof. For brevity's sake we write $u(x, t)$ instead of $[Q(t) w](x)$. First let us show $\partial u / \partial t$ belongs to $C\left(\left(0, t_{0}\right] ; C^{1}(\bar{D})\right)$. Differentiating (1. 1) by $t$, we formally obtain the linear equation

$$
\frac{\partial p}{\partial t}=L p+f_{u}(x, u(x, t)) p
$$

and the boundary condition

$$
\alpha p+(1-\alpha) \frac{\partial p}{\partial \nu}=0
$$

As $f_{u}(x, u(x, t))$ is Hölder continuous in $\bar{D} \times\left(0, t_{0}\right],(2.14)$ has a unique classical solution for any bounded continuous initial data at $t=t_{1}$, where $t_{1}$ is any given number in $\left(0, t_{0}\right]$. Set

$$
p\left(x, t_{1}\right)=\frac{\partial u\left(x, t_{1}\right)}{\partial t}
$$

(note that $\partial u / \partial t$ is bounded by virtue of the Hölder continuity of $f$ ). We claim that $p=\partial u / \partial t$ on $\bar{D} \times\left[t_{1}, t_{0}\right]$. To see this, we put

$$
\widetilde{u}(x, t)=u\left(x, t_{1}\right)+\int_{t_{1}}^{t} p(x, s) d s
$$

and prove $\widetilde{u}=u$. Since

$$
\begin{aligned}
L \widetilde{u} & =L u\left(x, t_{1}\right)+\int_{t_{1}}^{t} L p(x, s) d s \\
& =L u\left(x, t_{1}\right)+p-\frac{\partial u\left(x, t_{1}\right)}{\partial t}-\int_{t_{1}}^{t} f_{u}(x, u) p d s \\
& =p-f\left(x, u\left(x, t_{1}\right)\right)-\left[f_{u}(x, u) \widetilde{u}\right]_{t_{1}}^{t}+\int_{t_{1}}^{t} f_{u u}(x, u) \frac{\partial u}{\partial t} \widetilde{u} d s,
\end{aligned}
$$

it is not difficult to see that $w \equiv \widetilde{u}-u$ satisfies the equation 


$$
\frac{\partial w}{\partial t}=L w+f_{u}(x, u) w-\int_{t_{1}}^{t} f_{u u}(x, u) \frac{\partial u}{\partial t} w d s,
$$

as well as the boundary condition

$$
\alpha w+(1-\alpha) \frac{\partial w}{\partial \nu}=0
$$

Putting $\widetilde{w}=e^{-k t} w$, we get

$$
\left\{\begin{array}{l}
\frac{\partial \widetilde{w}}{\partial t}=L \widetilde{\widetilde{w}}-\left\{k-f_{u}(x, u)\right\} \widetilde{w}-\int_{t_{1}}^{t} e^{-k(t-s)} f_{u u} \frac{\partial u}{\partial t} \widetilde{w} d s \text { in } D \times\left[t_{1}, t_{0}\right] \\
\widetilde{w}\left(x, t_{1}\right)=0 \text { on } D \\
\alpha \widetilde{w}+(1-\alpha) \frac{\partial \widetilde{w}}{\partial \nu}=0 \text { on } \partial D \times\left[t_{1}, t_{0}\right],
\end{array}\right.
$$

where $k$ is a positive number so large as to satisfy the inequality

$$
k-f_{u}(x, u(x, t))>\int_{t_{1}}^{t_{0}}\left|f_{u u}(x, u) \frac{\partial u}{\partial t}\right| d s
$$

on $\bar{D} \times\left[t_{1}, t_{0}\right]$. By the boundary condition, $\widetilde{w}$ cannot take its positive maximum nor negative minimum anywhere on $\partial D \times\left[t_{1}, t_{0}\right]$. Now suppose $\widetilde{w} \not \equiv$, and let $\left(x^{\prime}, t^{\prime}\right)$ be the point where $|\widetilde{w}|$ takes its maximum. We can assume without loss of generality that $\widetilde{w}\left(x^{\prime}, t^{\prime}\right)>0$. Since $\left(x^{\prime}, t^{\prime}\right)$ $\in D \times\left(t_{1}, t_{0}\right]$, it follows that $\frac{\partial \widetilde{w}}{\partial t} \geqq 0, L \widetilde{w} \leqq 0$ and $\widetilde{w}>0$ at $\left(x^{\prime}, t^{\prime}\right)$. So

$$
\begin{aligned}
0 \leqq-\left\{k-f_{u}(x, u)\right\} \widetilde{w}\left(x^{\prime}, t^{\prime}\right)+\int_{t_{1}}^{t^{\prime}}\left|f_{u u} \frac{\partial u}{\partial t} \widetilde{w}\right| d s \\
\leqq-\left\{k-f_{u}(x, u)\right\} \widetilde{w}\left(x^{\prime}, t^{\prime}\right)+\widetilde{w}\left(x^{\prime}, t^{\prime}\right) \int_{t_{1}}^{t_{0}}\left|f_{u u} \frac{\partial u}{\partial t}\right| d s,
\end{aligned}
$$

which clealy contradicts (2.16). Therefore $\widetilde{w}$ must vanish everywhere, hence the equality $u=\widetilde{u}$ is established. As $t_{1}$ is any number in $\left(0, t_{0}\right]$, we get $\partial u / \partial t=p \in C\left(\left(0, t_{0}\right] ; C^{1}(\bar{D})\right)$.

Now we differentiate the form $J(u)$ by $t$. The following formal calculation is well-founded because $\partial u / \partial t$ belongs to $C\left(\left(0, t_{0}\right] ; C^{1}(\bar{D})\right)$ and $L u(=\partial u / \partial t-f(x, u))$ is bounded. By Green's formula,

$$
\begin{aligned}
\frac{d}{d t} \int_{D} & \left\{\frac{1}{2} \sum_{i, j} a_{i j} \frac{\partial u}{\partial x_{i}} \frac{\partial u}{\partial x_{j}}-F(x, u)\right\} d x \\
= & \int_{D}\left\{\sum_{i, j} a_{i j} \frac{\partial^{2} u}{\partial t \partial x_{i}} \frac{\partial u}{\partial x_{j}}-f(x, u) \frac{\partial u}{\partial t}\right\} d x
\end{aligned}
$$




$$
=\int_{\partial D} a \frac{\partial u}{\partial t} \frac{\partial u}{\partial \nu} d \widetilde{x}-\int_{D}\{L u+f(x, u)\} \frac{\partial u}{\partial t} d x .
$$

From the boundary condition of $u$ it follows that

$$
\begin{aligned}
\frac{\partial u}{\partial t} \frac{\partial u}{\partial \nu} & =\left(\alpha \frac{\partial u}{\partial t}\right) \frac{\partial u}{\partial \nu}+\frac{\partial u}{\partial t}\left\{(1-\alpha) \frac{\partial u}{\partial \nu}\right\} \\
& =-(1-\alpha) \frac{\partial}{\partial t}\left(\frac{\partial u}{\partial \nu}\right) \frac{\partial u}{\partial \nu}+\frac{\partial u}{\partial t}(-\alpha u+\beta) \\
& =-\frac{\partial}{\partial t}\left\{\frac{1-\alpha}{2}\left(\frac{\partial u}{\partial \nu}\right)^{2}+\frac{\alpha}{2} u^{2}-\beta u\right\} .
\end{aligned}
$$

Therefore

$$
\frac{d}{d t} J(u)=-\int_{D}\{L u+f(x, u)\} \frac{\partial u}{\partial t} d x=-\int_{D}\left(\frac{\partial u}{\partial t}\right)^{2} d x \text {. Q.E.D. }
$$

Let $\psi$ be an element of $\operatorname{Dom}(Q(\infty))\left(\equiv \bigcap_{t>0} \operatorname{Dom}(Q(t))\right)$. Looking upon the set $\{Q(t) \phi\}_{0<t<\infty}$ as an orbit described in some function space, we can define its $\omega$-limiting set in the usual way-as is usual in the theory of dynamical systems:

Definition 1. For any $\psi \in \operatorname{Dom}(Q(\infty))$ the $\omega$-limiting set of $\psi$ in a function space $X$ is defined as follows:

$$
\Omega(X \mid \psi)=\bigcap_{t>0} c l_{X}\left(\{Q(\tau) \psi\}_{\tau \geq t}\right),
$$

where $c l_{X}(\cdot)$ denotes the closure of a set in $X$. (We understand that $\Omega(X \mid \psi)=\varnothing$ if $\psi$ belongs to $\mathscr{B}^{0}(D) \backslash \operatorname{Dom}(Q(\infty))$, i.e., if the solution of (1.1) with initial data $\psi$ blows up in some finite time interval.)

Of importance to us is the case where $X=C^{1}(\bar{D}) \cap C^{2}(D)$, but we also consider the case where $X=L^{\infty}(D)$ with the weak* topology. As $L^{1}(D)$ is separable, $L^{\infty}(D)$-weak* satisfies the first countability axiom. Therefore, in either of these cases a function $\varphi(x)$ belongs to $\Omega(X \mid \psi)$ if and only if there exists a sequence $0<t_{1}<t_{2} \cdots \rightarrow \infty$ such that

$$
\lim _{m \rightarrow \infty} Q\left(t_{m}\right) \psi=\varphi \quad \text { in } X .
$$

The continuous embeddinss $C^{1}(\bar{D}) \cap C^{2}(D) \subset C(\bar{D}) \subset L^{\infty}(D)-w_{\text {.* }}$ 
yield

$$
\Omega\left(C^{1}(\bar{D}) \cap C^{2}(D) \mid \psi\right) \subset \Omega(C(\bar{D}) \mid \psi) \subset \Omega\left(L^{\omega}(D)-w . * \mid \psi\right) .
$$

As a matter of fact, these apparently different $\omega$-limiting sets coincide with each other as is seen in the following theorem:

Theorem 2.8. Let (A) hold and let $\psi \in \operatorname{Dom}(Q(\infty))$. Then

i) $\Omega\left(C^{1}(\bar{D}) \cap C^{2}(D) \mid \psi\right)=\Omega\left(L^{\infty}(D)-w . * \mid \psi\right)$;

ii) it consists of solutions of (1.2);

iii) it is not empty if there exists a sequence $0<t_{1}<t_{2}<\cdots \rightarrow \infty$ such that $Q\left(t_{m}\right)$ \& remains bounded in $L^{\infty}(D)$ as $m \rightarrow \infty$.

Proof. We first show iii). For simplicity let $u_{m}(x)$ denote $\left[Q\left(t_{m}\right) \psi\right](x)$ for $m=1,2, \cdots$. As $\left\{u_{m}\right\}_{m \in \mathbf{N}}$ is bounded in $\mathscr{B}^{0}(D)$, it follows from Theorem 2.4 that $\left\{Q u_{m}\right\}_{m \in \boldsymbol{N}}$ is relatively compact in $C\left(\left(0, t_{0}\right] ; C^{1}(\bar{D}) \cap C^{2}(D)\right)$ for some $t_{0}>0$. So $\left\{Q\left(t_{0}\right) u_{m}\right\}_{m \in \mathbf{N}} \equiv\left\{\underline{Q}\left(t_{m}\right.\right.$ $\left.\left.+t_{0}\right) \phi\right\}_{m \in N}$ is relatively compact in $C^{1}(\bar{D}) \cap C^{2}(D)$, hence the conclusion.

Secondly we prove i) and ii) at one time. Suppose $\Omega\left(L^{\infty}(D)\right.$ $-w \cdot * \mid \psi)$ is not empty and let $\varphi$ be any element of it. What we have to show is that $\varphi$ belongs to $\Omega\left(C^{1}(\bar{D}) \cap C^{2}(D) \mid \psi\right)$ and that it is a solution of (1.2). By definition, there exists a sequence $0<t_{1}<t_{2}<\cdots \rightarrow \infty$ such that

$$
\lim _{m \rightarrow \infty} Q\left(t_{m}\right) \psi=\varphi \quad \text { in } \quad L^{\omega}(D) \text {-weak*. }
$$

Using Banach-Steinhaus theorem, we see that $u_{m} \equiv Q\left(t_{m}\right) \psi$ remains bounded in $L^{\infty}(D)$ as $m \rightarrow \infty$. So, with a suitable positive number $t_{0}$ as above, $\left\{Q u_{m}\right\}_{m \in \boldsymbol{N}}$ is bounded in $L^{\infty}\left(D \times\left(0, t_{0}\right)\right)$ and relatively compact in $C\left(\left(0, t_{0}\right] ; C^{1}(\bar{D}) \cap C^{2}(D)\right)$. Since $J(Q(t) \psi)$ is monotone decreasing in $t$ by virtue of Lemma 2.7,

$$
J\left(u_{1}\right) \geqq J\left(u_{m}\right),
$$

hence

$$
\begin{aligned}
\int_{D} \sum_{i, j} a_{i j} \frac{\partial u_{m}}{\partial x_{i}} \frac{\partial u_{m}}{\partial x_{j}} d x+\int_{\partial D} a(1-c)\left(\frac{\partial u_{m}}{\partial \nu}\right)^{2} d \widetilde{x} \\
\quad \leqq 2 J\left(u_{1}\right)+2 \int_{D} F\left(x, u_{m}\right) d x+\int_{\partial D} a\left(2 \beta u_{m}-\alpha u_{m}{ }^{2}\right) d \widetilde{x},
\end{aligned}
$$


for $m=1,2, \cdots$. The right-hand side of this inequality is bounded with respect to $m$ because of the boundedness of $\left\{u_{1}, u_{2}, \cdots\right\}$ in the maximum norm. Considering that the matrix $\left(a_{i j}(x)\right)$ is uniformly positive definite and taking into account the non-negativity of $a(1-\alpha)\left(\partial u_{m} / \partial \nu\right)^{2}$, we find that $u_{m}$ remains bounded in $H^{1}(D)^{1)}$ as $m \rightarrow \infty$. So $\left\{u_{m}\right\}_{m \in \boldsymbol{N}}$ is relatively compact in $L^{2}(D)$, since $D$ is a bounded domain. Recall that $u_{m}$ converges to $\varphi$ as $m \rightarrow \infty$ in $L^{\infty}(D)-w . *$. It follows that this convergence also occurs in $L^{2}(D)$, hence, by Lemma 2.6 ii), there exists a function $h(x, t)$ such that

$$
\lim _{m \rightarrow \infty} \sup _{0<t \leqq t_{0}}\left\|Q(t) u_{m}-h(x, t)\right\|_{L^{2}(D)}=0 .
$$

Combining (2.17) and the fact that $\left\{Q u_{m}\right\}_{m \in \boldsymbol{N}}$ is relatively compact in $C\left(\left(0, t_{0}\right] ; C^{1}(\bar{D}) \cap C^{2}(D)\right)$, we see, under a suitable modification of $h$ on a null set, that given any $\delta \in\left(0, t_{0}\right]$ and any compact set $K \subset D$

$$
\begin{aligned}
& \lim _{m \rightarrow \infty} Q(t) u_{m}=h(x, t) \quad \text { in } \quad C^{2}(K), \\
& \lim _{m \rightarrow \infty} Q(t) u_{m}=h(x, t) \text { in } \quad C^{1}(\bar{D}),
\end{aligned}
$$

uniformly in $\delta \leqq t \leqq t_{0}$.

On the other hand, using Lemma 2.7 again, we get

$$
\int_{t_{1}+t_{0}}^{t_{m}+t_{0}} d \tau \int_{D}\left\{\frac{\partial}{\partial t}[Q(\tau) \psi](x)\right\}^{2} d x=J\left(\underline{Q}\left(t_{0}\right) u_{1}\right)-J\left(\underline{Q}\left(t_{0}\right) u_{m}\right) .
$$

Since $\left\{Q\left(t_{0}\right) u_{m}\right\}$ is a bounded set in $C^{1}(\bar{D}), J\left(Q\left(t_{0}\right) u_{m}\right)$ remains bounded as $m \rightarrow \infty$. Therefore

$$
\int_{t_{1}+t_{0}}^{\infty} d \tau \int_{D}\left\{\frac{\partial}{\partial t} Q(\tau) \phi\right\}^{2} d x<\infty
$$

Using (2.18) and (2.20) we obtain

$$
\begin{aligned}
0 & =\lim _{m \rightarrow \infty} \int_{t_{m}+\delta}^{t_{m}+t_{0}} d \tau \int_{D}\left\{\frac{\partial}{\partial t} Q(\tau) \phi\right\}^{2} d x \\
& =\lim _{m \rightarrow \infty} \int_{\delta}^{t_{0}} d \tau \int_{D}\left\{\frac{\partial}{\partial t} Q(\tau) u_{m}\right\}^{2} d x
\end{aligned}
$$

1) $H^{1}(D)$ denotes the Banach space of all functions with finite norm $\left\{\int_{D} w^{2} d x+\int_{D}|\nabla w|^{2} d x\right\}^{1 / 2}$ 


$$
\begin{aligned}
& \geqq \lim _{m \rightarrow \infty} \int_{\delta}^{t_{0}} d \tau \int_{K}\left\{\frac{\partial}{\partial t} Q(\tau) u_{m}\right\}^{2} d x \\
& =\lim _{m \rightarrow \infty} \int_{\delta}^{t_{0}} d \tau \int_{K}\left\{L Q(\tau) u_{m}+f\left(x, Q(\tau) u_{m}\right)\right\}^{2} d x \\
& =\int_{\delta}^{t_{0}} d \tau \int_{K}\{L h(x, \tau)+f(x, h(x, \tau))\}^{2} d x,
\end{aligned}
$$

for any $\delta \in\left(0, t_{0}\right]$ and any compact set $K \subset D$, and hence

$$
L h(x, t)+f(x, h(x, t))=0 \quad \text { in } \quad D \times\left(0, t_{0}\right] .
$$

It also follows that

$$
\frac{\partial h}{\partial t}(x, t)=0 \quad \text { in } \quad D \times\left(0, t_{0}\right]
$$

since $u_{m} \rightarrow h$ and $\partial u_{m} / \partial t \rightarrow 0$ (as $m \rightarrow \infty$ ) uniformly on any $K \times\left[\delta, t_{0}\right]$. Meanwhile it follows from (2.19) and the boundary condition of $Q(t) u_{m}$ that

$$
\alpha h(x, t)+(1-\alpha) \frac{\partial h}{\partial \nu}(x, t)=0 \quad \text { on } \quad \partial D \times\left(0, t_{0}\right]
$$

Combining (2.21), (2.22) and (2.23), we conclude that $h$ is independent of $t$ and is a solution of (1.2) regarded as a function of $x$. Moreover it belongs to $\Omega\left(C^{1}(\bar{D}) \cap C^{2}(D) \mid \psi\right)$ because of (2.18) and (2.19). Passing to the limit in the equality $Q(t) u_{m}=T(t) u_{m}+S(t) f\left(x, Q(t) u_{m}\right)$ and using (2.17) and Lemma 2.6 i), we get

$$
h(x, t)=[T(t) \varphi](x)+[S(t) f(x, h)](x) .
$$

Consequently

$$
\lim _{t \rightarrow 0} h=\lim _{t \rightarrow 0} T(t) \varphi=\varphi \quad \text { in } \quad L^{2}(D)
$$

(see [8; Theorem 6] for the latter equality). It implies $\varphi(x)=h\left(x, t_{0}\right)$ for a.e. $x \in D$, hence $\varphi$ is a solution of (1.2) and belongs to $\Omega\left(C^{1}(\bar{D})\right.$ $\left.\cap C^{2}(D) \mid \psi\right)$.

Q.E.D.

Owing to this theorem, there is no need to distinguish $\Omega(X \mid \psi)$ from $\Omega\left(C^{1}(\bar{D}) \cap C^{2}(D) \mid \psi\right)$ so long as we have the continuous embeddings

$$
C^{1}(\bar{D}) \cap C^{2}(D) \subset X \subset L^{\infty}(D)-\text { weak }^{*} .
$$


So hereafter we adopt the abridged notation

$$
\Omega(\psi) \equiv \Omega(X \mid \psi),
$$

where $X$ is any space satisfying the above condition.

Theorem 2.9. Let (A) hold, let $\psi \in \operatorname{Dom}(Q(\infty))$, and let $Y$ be a nonempty subset of $\Omega(\psi)$ such that $Y$ and $\Omega(\psi) \backslash Y$ are closed in $C^{1}(\bar{D}) \cap C^{2}(D)$. Suppose $Y$ is bounded in $L^{\infty}(D)$. Then $\Omega(\psi)=Y$ and, given any neighborhood $W$ of $Y$ in $C^{1}(\bar{D}) \cap C^{2}(D)$, there exists a finite number $T \geqq 0$ such that

$$
\{Q(t) \psi\}_{t \leqq T} \subset W
$$

Proof. We have only to prove the latter part, for the former follows immediately from the latter. Let

$$
V(\varepsilon)=\left\{w \in \mathscr{B}^{0}(D) ;\|w-\varphi\|_{L^{\infty}(D)}<\varepsilon \text { for some } \varphi \in Y\right\} .
$$

As $Y$ is bounded in $L^{\infty}(D)$, so is $V(\varepsilon)$ for each fixed $\varepsilon>0$. Hence, by virtue of Corollary 2.5, $V(1) \cap \Omega(\psi)$ is relatively compact in $C^{1}(\bar{D})$ $\cap C^{2}(D)$. So the topology induced from $C^{1}(\bar{D}) \cap C^{2}(D)$ and the one from $L^{\infty}(D)$ are equivalent on this set. Considering that $Y$ is compact and is separated by an open set in $C^{1}(\bar{D}) \cap C^{2}(D)$ from $\Omega(\psi) \backslash Y$, we find that there exists an $\varepsilon_{0} \in(0,1]$ such that

$$
V\left(\varepsilon_{0}\right) \cap \Omega(\psi)=Y \text {. }
$$

Now we claim that for any $0<\varepsilon<\varepsilon_{0}$ there exists a $T^{\prime}>0$ such that

$$
\{\underline{Q}(t) \psi\}_{t \geqq T^{\prime}} \subset V(\varepsilon) \text {. }
$$

Suppose (2.26) does not hold. Then there exist an $\varepsilon_{1} \in\left(0, \varepsilon_{0}\right)$ and a sequence $0<t_{1}<t_{2}<\cdots \rightarrow \infty$ such that

$$
Q\left(t_{2 m-1}\right) \psi \in V\left(\frac{\varepsilon_{1}}{2}\right)
$$

$$
Q\left(t_{2 m}\right) \psi \notin V\left(\varepsilon_{1}\right)
$$

for $m=1,2, \cdots$. By the way, for any $\varphi \in Y$ and $w \in V\left(\varepsilon_{1} / 2\right), \bar{u}=Q(t) w$ $-\varphi$ satisfies the reduced equation 


$$
\frac{\partial \bar{u}}{\partial t}=L \bar{u}+\{f(x, \bar{u}+\varphi(x))-f(x, \varphi(x))\},
$$

as well as the boundary condition

$$
\alpha \bar{u}+(1-\alpha) \frac{\partial \bar{u}}{\partial \nu}=0
$$

So it is not difficult to see that there exists a $t_{0}>0$ such that

$$
Q(t) w \in V\left(\varepsilon_{1}\right), \quad 0 \leqq t \leqq t_{0},
$$

for all $w \in V\left(\varepsilon_{1} / 2\right)$ (see the proof of Theorem 2.4i)). Combining (2.27) and (2.28), we find that for each $m=1,2, \cdots$ there exists a $t_{m}{ }^{\prime} \in\left[t_{2 m-1}, t_{2 m}\right)$ such that

$$
Q\left(t_{m}^{\prime}+t\right) \phi \in V\left(\varepsilon_{1}\right) \backslash V\left(\frac{\varepsilon_{1}}{2}\right), \quad 0 \leqq t \leqq t_{0} .
$$

Consequently, by virtue of Theorem 2.4 ii), $\left\{Q\left(t_{m}{ }^{\prime}+t_{0}\right) \psi\right\}_{m=1.2, \ldots}$ is relatively compact in $C^{1}(\bar{D}) \cap C^{2}(D)$. It implies that $\Omega(\psi)$ meets with the closure of $V\left(\varepsilon_{1}\right) \backslash V\left(\varepsilon_{1} / 2\right)$, hence $\Omega(\psi) \backslash Y$ meets with $V\left(\varepsilon_{0}\right)$, a contradiction to (2.25). Thus (2.26) is established.

As a result of $(2.26),\{Q(t) \psi\}_{t \geqq o}$ is relatively compact in $C^{1}(\bar{D})$ $\cap C^{2}(D)$ for any $\delta>0$. It can be seen from the boundedness of $\{Q(t) \phi\}_{t \geq 0}$ in $L^{\infty}(D)$, Theorem 2.4 ii) and the fact that

$$
\{Q(t) \psi)_{t \geqq o}=\left\{Q(\delta) w ; w \in\{Q(t) \psi\}_{t \geqq 0}\right\} .
$$

Accordingly the topology of $C^{1}(\bar{D}) \cap C^{2}(D)$ and that of $L^{\infty}(D)$ are equivalent on the closure of $\{Q(t) \phi\}_{t \geq \delta}$, hence, combining this fact and (2.26), we obtain the conclusion.

Q.E.D.

Corollary 2.10. Let (A) hold and let $\psi \in \operatorname{Dom}(Q(\infty))$. Suppose $Q(t) \psi$ remains bounded in the $L^{\infty}(D)$ norm as $t \rightarrow \infty$. Then $\Omega(\psi)$ is a non-empty connected set in $C^{1}(\bar{D}) \cap C^{2}(D)$.

Corollary 2.11. Let (A) hold and let $\phi \in \operatorname{Dom}(Q(\infty))$. Suppose $\Omega(\psi)$ contains an isolated point $v$ (reith respect to the topology of $\left.C^{1}(\bar{D}) \cap C^{2}(D)\right)$. Then $\Omega(\psi)=\{v\}$ and

$$
\lim _{t \rightarrow \infty} Q(t) \psi=v \quad \text { in } \quad C^{1}(\bar{D}) \cap C^{2}(D) .
$$


These corollaries are direct consequences of Theorems 2.9 and 2.8 iii).

\section{§ 3. Strong Instability of Equilibrium Solutions}

A solution $v$ of (1.2) is said to be stable (in the maximum norm) if given any $\varepsilon>0$ there exists a $\delta>0$ such that

$$
\|Q(t) \phi-v\|_{L^{\infty}(D)}<\varepsilon, \quad 0<t<\infty,
$$

for any $\phi \in \mathscr{B}^{0}(D)$ satisfying $\|\psi-v\|_{L^{\infty}(D)}<\delta$, where $Q$ is what is introduced in (2.8). We say $v$ is unstable if $v$ is not stable. As we are concerned with real-valued solutions, it is possible, and convenient as well, to discriminate between upward instability and downward one. It is also convenient to introduce the notion of instability in a stronger sense.

Definition 2. A solution $v$ of (1.2) is unstable upward if there exists an $\varepsilon_{0}>0$ such that for any $\delta>0$ there exists a function $\psi \in \mathscr{B}^{0}(D)$ which satisfies $v(x) \leqq \psi(x) \leqq v(x)+\delta$ everywhere on $D$ and also the inequality

$$
\left[Q\left(t_{0}\right) \psi\right]\left(x_{0}\right) \geqq v\left(x_{0}\right)+\varepsilon_{0}
$$

at some $\left(x_{0}, t_{0}\right)$. We say $v$ is strongly unstable upward if there exists an $\varepsilon_{0}>0$ such that given any $\psi \in \mathscr{B}^{\circ}(D)$ satisfying $\psi \geqq v$ and $\psi \not \equiv v$ (3.1) holds at some $\left(x_{0}, t_{0}\right)$. By reversing the inequalities, downward instability in either sense is defined likewise.

It is easy to see, by virtue of the comparison theorem, that $v$ is stable in the maximum norm if and only if it is unstable neither upward nor downward.

Before presenting our results we introduce the following notation:

$$
\begin{aligned}
& X^{+}(v)=\left\{w \in \mathscr{B}^{0}(D) ; w \geqq v, w \neq v\right\}, \\
& X^{-}(v)=\left\{w \in \mathscr{B}^{0}(D) ; w \leqq v, w \neq v\right\}, \\
& S^{+}(v)=S \cap X^{+}(v), \\
& S^{-}(v)=S \cap X^{-}(v),
\end{aligned}
$$

where $S$ is the set of all the solutions of (1.2). 
Here we are ready to state the most important theorem in this chapter:

Theorem 3. 1. Let (A) hold (see § 2) and let $v$ be a solution of (1.2) which is strongly unstable upward.

i) Suppose $S^{+}(v) \neq \varnothing$. Then it has the minimum of it, i.e., there exists a solution $v^{+} \in S^{+}(v)$ such that

$$
v^{+}(x) \leqq \widetilde{v}(x) \text { on } \bar{D}
$$

for all $\widetilde{v} \in S^{+}(v)$. Moreover, for any $\phi \in X^{+}(v) \cap X^{-}\left(v^{+}\right)$,

$$
\lim _{t \rightarrow \infty} Q(t) \psi=v^{+}
$$

in the topology of $C^{1}(\bar{D}) \cap C^{2}(D)$.

ii) Suppose $S^{+}(v)=\varnothing$. Then, given any $\psi \in X^{+}(v)$, there exists a $T, 0<T \leqq \infty$, such that

$$
\lim _{t \rightarrow T} \max _{x \in \bar{D}}[Q(t) \psi](x)=+\infty .
$$

Remark 3.2. A similar proposition holds true for $S^{-}(v)$ and its maximum $v^{-}$if $v$ is strongly unstable downward.

The proof shall be given later in this section. This theorem will find its wider application when coupled with one of the following theorems which are more or less familiar to us.

Theorem 3. 3. Let (A) hold, let $v$ be a solution of (1.2), and let $\lambda_{1}$ be the least eigenvalue of the following eigenvalue problem:

$$
\begin{aligned}
& \widetilde{L} \varphi+\lambda \varphi=0 \quad \text { in } \quad D, \\
& \alpha \varphi+(1-\alpha) \frac{\partial \varphi}{\partial \nu}=0 \quad \text { on } \partial D,
\end{aligned}
$$

where

$$
\widetilde{L} \varphi \equiv L \varphi+f_{u}(x, v(x)) \varphi .
$$

If $\lambda_{1}<0, v$ is strongly unstable both upward and dorenward.

Next theorem requires a further assumption on the boundary condition. 
(B) Either 1) or 2) holds:

1) $\alpha \equiv 1$ on $\partial D$.

2) $1-\alpha$ never vanishes on $\partial D$.

Theorem 3.4. Let (A), (B) hold and let $v$ be a solution of (1.2). If $v$ is unstable upward (resp. downward), it is strongly unstable upward (resp. downward).

As a consequence of Theorems 3.1 and 3.4 we have

Corollary 3. 5. Let (A), (B) hold and let $v$ be a solution of (1.2). Suppose one of the following conditions holds. Then $v$ is stable from above, i.e., not unstable upward.

1) There exists a function $\phi \in X^{+}(v)$ such that $Q(t) \psi$ converges to $v$ as $t \rightarrow \infty$ (for instance in the maximum norm).

2)

$$
\inf _{\hat{v} \in S^{+}(v)} \widetilde{\widetilde{v}}(x)=v(x) \text { on } \bar{D} .
$$

Of course an analogous assertion holds good for the stability from below.

Some applications of Theorem 3.1 in combination with Theorem 3.4 will be found in $\S 4$ and $\S 6$, while in $\S 5$ are those of Theorem 3.1 in combination with Theorem 3. 3 .

Now let us begin the proof of these results.

Lemma 3. 6. Let (A) hold and let $v$ be a solution of (1.2). Suppose $v$ is strongly unstable upward. Then

i) $v$ is isolated from $S^{+}(v)$ in the maximum norm;

ii) for any $\psi \in X^{+}(v)$,

$$
\Omega(\psi) \subset S^{+}(v)
$$

$(\operatorname{See}(2.24)$.

Proof. i) Let $\varepsilon_{0}$ be as in Definition 2 and let $V=\left\{w \in \mathscr{B}^{\circ}(D)\right.$; $\left.\|w-v\|_{\infty}<\varepsilon_{0}\right\}$. It follows from the definition of $\varepsilon_{0}$ that $V \cap X^{+}(v)$ does not contain any equilibrium solutions. So $V \cap S^{+}(v)=\varnothing$. 
ii) We assume $\Omega(\psi) \neq \varnothing$, for otherwise the statement is trivial. By virtue of the comparison Theorem and Theorem $2.8 \mathrm{ii}), \Omega(\psi)$ is contained in $S^{+}(v) \cup\{v\}$. Since $v$ is an isolated point of $S^{+}(v) \cup\{v\}$, it follows from Corollary 2.11 that either $\Omega(\psi)=\{v\}$ or $\Omega(\psi) \subset S^{+}(v)$. Suppose that the former holds. Then we see, from Corollary 2.11 again, that $Q(t) \psi$ converges to $v$ in $C^{1}(\bar{D}) \cap C^{2}(D)$, which contradicts the strong upward instability of $v$. Therefore the former cannot hold, hence the conclusion.

Q.E.D.

We introduce into $S^{+}(v)$ (resp. $S^{-}(v)$ ) an order relation defined as follows:

$v_{1} \geqq v_{2}$ means $v_{1}(x) \geqq v_{2}(x)$ everywhere on $\bar{D} . \quad S^{+}(v)$ (resp. $S^{-}(v)$ ) is then regarded as a partially ordered set with respect to this relation.

Lemma 3. 7. Let (A) hold and let $v$ be a solution of (1.2). Suppose $v$ is strongly unstable upward and that $S^{+}(v) \neq \varnothing$. Then for any $v_{1}, \quad v_{2} \in S^{+}(v)$ there exists the greatest lower bound of $\left\{v_{1}, v_{2}\right\}$ in the ordered set $S^{+}(v)$.

Proof. Put

$$
h(x)=\min \left\{v_{1}(x), v_{2}(x)\right\}
$$

By virtue of the strong maximum principle, $h(x)>v(x)$ on $D$ (cf. Lemmas 3.8 and 3.9). Hence $h \in X^{+}(v)$ and, by the comparison theorem,

$$
v \leqq Q(t) h \leqq v_{i}, \quad i=1,2,
$$

for all $t \geqq 0$. Applying Corollary 2.10 and Lemma 3.6 ii) to $h$, we find that $\Omega(h)$ is a non-emply subset of $S^{+}(v)$. The above inequalities thus imply that $\left\{v_{1}, v_{2}\right\}$ has at least one lower bound in $S^{+}(v)$. Let $\widetilde{v} \in S^{+}(v)$ be any lower bound of $\left\{v_{1}, v_{2}\right\}$. Since $\widetilde{v} \leqq h$,

$$
\widetilde{v} \leqq Q(t) h \leqq v_{i}, \quad i=1,2,
$$

for all $t \geqq 0$. So any element of $\Omega(h)$ is an upper bound of $\{\widetilde{v}\}$. It is now easy to see that $\Omega(h)$ contains only one element, namely the greatest lower bound of $\left\{v_{1}, v_{2}\right\}$.

Q.E.D. 
It follows from this lemma that any finite subset of $S^{+}(v)$ has the greatest lower bound of it in $S^{+}(v)$. We denote by $v_{1} \wedge v_{2} \wedge \cdots \wedge v_{m}$ the greatest lower bound of $\left\{v_{1}, v_{2}, \cdots, v_{m}\right\}$.

Proof of Theoren 3.1. i) We merely show the existence of the minimum $v^{+}$. The rest of i) then follows immediately, if the comparison theorem, Theorem 2.8 iii), Lemma 3. 6 ii) and Corollary 2. 11 are taken into account.

Put

$$
\Psi(x)=\inf _{\tilde{v} \in S^{+}(v)} \widetilde{v}(x), \quad x \in \bar{D} .
$$

What we have to show now is $\Psi \in S^{+}(v)$. Take an element $v_{0}$ of $S^{+}(v)$ and fix it. Then

$$
\Psi(x)=\inf _{\tilde{v} \in S^{+} \wedge v_{0}} \widetilde{v}(x),
$$

where $S^{+} \wedge v_{0}$ denotes $\left\{\widetilde{v} \wedge v_{0} ; \widetilde{v} \in S^{+}(v)\right\}$. So, given any $\varepsilon>0$ and any finite number of points $x_{1}, \cdots, x_{k} \in \bar{D}$, there exist $v_{1}, \cdots, v_{k} \in S^{+} \wedge v_{0}$ such that

$$
\Psi\left(x_{i}\right) \leqq v_{i}\left(x_{i}\right) \leqq \Psi\left(x_{i}\right)+\varepsilon
$$

for $i=1, \cdots, k$. Hence

$$
\Psi\left(x_{i}\right) \leqq v_{1} \wedge \cdots \wedge v_{k}\left(x_{i}\right) \leqq \Psi\left(x_{i}\right)+\varepsilon
$$

for $i=1, \cdots, k$. It follows that $\Psi$ is an accumulation point of $S^{+} \wedge v_{0}$ in the topology of pointwise convergence on $\bar{D}$. On the other hand, since $S^{+} \wedge v_{0}$ is bounded by $v$ from below and by $v_{0}$ from above, $S^{+} \wedge v_{0}$ is relatively compact in $C^{1}(\bar{D}) \cap C^{2}(D)$ by virtue of Corollary 2.5. Consequently $\Psi$ is also an accumulation point of $S^{+} \wedge v_{0}$ in $C^{1}(\bar{D}) \cap C^{2}(D)$, therefore $\Psi$ is a solution of (1.2). This means that $\Psi$ belongs to $S^{+}(v) \cup\{v\}$, and as $v$ is isolated from $S^{+}(v)$ by Lemma 3.6 i), $\Psi$ must belong to $S^{+}(v)$. Thus the statement i) is established.

ii) Let $T=\sup \{t>0 ; \phi \in \operatorname{Dom}(Q(t))\}$, and suppose there exist a constant $M>0$ and a sequence $0<t_{1}<t_{2}<\cdots \rightarrow T$ such that

$$
\sup _{x \in \bar{D}}\left[Q\left(t_{m}\right) \psi\right](x) \leqq M
$$

for $m=1,2, \cdots$. As $Q(t) \psi$ is bounded by $v$ from below, it then follows 
that $\left\{Q\left(t_{m}\right) \psi\right\}_{m \in \boldsymbol{N}}$ is bounded in $L^{\infty}(D)$. Here we have two cases, i.e., the case where $T$ is a finite number and the case where $T=\infty$. In the first case, on applying Theorem 2.4 i) to $B=\left\{Q\left(t_{m}\right) \psi\right\}_{m \in \mathbf{N}}$, we are led to a contradiction. In the second case, an application of Theorem 2.8 iii) yields $\Omega(\psi) \neq \varnothing$. So it follows from Lemma 3.6 ii) that $S^{+}(v)$ $\neq \varnothing$, which contradicts the presupposition. Thus, in either case, our supposition (3. 3) has turned out false.

Q.E.D.

Proof of Theorem 3.3. Let $\varphi_{1}$ be one of the eigenfunctions of (3. 2) belonging to $\lambda_{1}$. It is well-known that $\varphi_{1}$ never changes sign in $D$ (a consequence of Krein-Rutman Theory). So we can assume in the sequel that $\varphi_{1}>0$ in $D$ and that $\left\|\varphi_{1}\right\|_{L^{2}(D)}=1$. Since $\lambda_{1}<0$, there exists an $\varepsilon_{0}>0$ such that

$$
f(x, v(x)+\varepsilon)-f(x, v(x)) \geqq \varepsilon\left\{f_{u}(x, v(x))+\frac{\lambda_{1}}{2}\right\}
$$

for all $0 \leqq \varepsilon \leqq \varepsilon_{0}$ and $x \in \bar{D}$.

Now suppose that some function $\psi \in X^{+}(v)$ satisfies the inequality

$$
\|Q(t) \psi-v\|_{L^{\infty}(D)} \leqq \varepsilon_{0}
$$

for all $t \geqq 0$. By Green's formula,

$$
\begin{aligned}
\frac{d}{d t}\left\langle Q(t) \psi-v, \varphi_{1}\right\rangle=\left\langle\frac{\partial}{\partial t} Q(t) \psi, \varphi_{1}\right\rangle \\
=\left\langle L Q(t) \psi+f(x, Q(t) \psi), \varphi_{1}\right\rangle \\
=\left\langle L\{Q(t) \psi-v\}+\{f(x, Q(t) \psi)-f(x, v)\}, \varphi_{1}\right\rangle, \\
\quad=\left\langle Q(t) \psi-v, L \varphi_{1}\right\rangle+\left\langle f(x, Q(t) \psi)-f(x, v), \varphi_{1}\right\rangle,
\end{aligned}
$$

where $\langle$,$\rangle denotes the inner product on L^{2}(D)$. Using (3.4) and (3. 5), we get

$$
\frac{d}{d t}\left\langle Q(t) \psi-v, \varphi_{1}\right\rangle \geqq-\frac{\lambda_{1}}{2}\left\langle Q(t) \psi-v, \varphi_{1}\right\rangle,
$$

therefore

$$
\left\langle Q(t) \psi-v, \varphi_{1}\right\rangle \geqq \exp \left\{-\frac{\lambda_{1}}{2} t\right\}\left\langle\psi-v, \varphi_{1}\right\rangle
$$

As $\lambda_{1}<0$ and $\left.\left\langle\psi-v, \varphi_{1}\right\rangle\right\rangle 0$, it follows that 


$$
\lim _{t \rightarrow \infty}\left\langle Q(t) \psi-v, \varphi_{1}\right\rangle=\infty,
$$

which contradicts (3.5) because $D$ is bounded. So there exists no such a function $\psi \in X^{+}(v)$ as to satisfy (3.5). Hence the strong upward instability of $v$. The strong downward instability can be shown likewise.

Q.E.D.

Lemma 3. 8. Let (A), (B1) hold and let $v$ be a solution of (1.2). Given any $\psi \in X^{+}(v)$ and any $t_{1}>0$ such as $\psi \in \operatorname{Dom}\left(Q\left(t_{1}\right)\right)$, there exists a constant $c>0$ such that

$$
Q\left(t_{1}\right) \psi \geqq v+c \rho,
$$

where $\rho(x)$ is the distance between $x$ and $\partial D$.

Proof. Put

$$
M=\sup _{0 \leq t \leq t_{1}}\left\|f_{u}(x, Q(t) \phi)\right\|_{L^{\infty}(D)}
$$

and let us consider the initial-boundary value problem

$$
\left\{\begin{array}{l}
\frac{\partial \bar{u}}{\partial t}=L \bar{u}-M \bar{u} \\
\bar{u}(x, 0)=\psi(x)-v(x) \\
\alpha \bar{u}+(1-\alpha) \frac{\partial \bar{u}}{\partial \nu}=0 .
\end{array}\right.
$$

By virtue of the comparison theorem we have

$$
\text { (3.7) } Q\left(t_{1}\right) \psi-v \geqq \bar{u}\left(x, t_{1}\right)=e^{-M t_{1}} \int_{D} U\left(x, y, t_{1}\right)\{\psi(y)-v(y)\} d y \text {, }
$$

where $U$ is the fundamental solution of the equation $\partial u / \partial t=L u$ with the same boundary condition as above. Since

$$
\begin{array}{ll}
U\left(x, y, t_{1}\right)>0 & \text { for }(x, y) \in D \times D, \\
\frac{\partial U}{\partial \nu}\left(x, y, t_{1}\right)<0 & \text { for }(x, y) \in \partial D \times D
\end{array}
$$

(see $[8], \S 9$ ), and as $\psi-v$ is non-negative and is not identical to zero, it follows that 


$$
\begin{aligned}
& \bar{u}\left(x, t_{1}\right)>0 \quad \text { in } D, \\
& \frac{\partial \bar{u}}{\partial \nu}\left(x, t_{1}\right)<0 \text { on } \partial D .
\end{aligned}
$$

So we can easily find that there exists a positive constant $c$ such that

$$
\bar{u}\left(x, t_{1}\right) \geqq c \rho,
$$

hence we obtain the conclusion.

Q.E.D.

Lemma 3.9. Let (A), (B2) hold and let $v$ be a solution of (1.2). Given any $\phi \in X^{+}(v)$ and any $t_{1}>0$ such as $\phi \in \operatorname{Dom}\left(Q\left(t_{1}\right)\right)$, there exists a constant $c>0$ such that

$$
Q\left(t_{1}\right) \psi \geqq v+c
$$

everyzhere on $\bar{D}$.

The proof is just similar to the previous one except that we use here the following property of $U$ :

$$
U\left(x, y, t_{1}\right)>0 \quad \text { on } \quad \bar{D} \times \bar{D} .
$$

Proof of Theorem 3.4. First we prove this theorem in the case where $(\mathbf{A}),(\mathbf{B 1})$ hold. Let $\varepsilon_{0}$ be as in the definition of the upward instability of $v$ (see Definition 2) and let $\psi$ be any element of $X^{+}(v)$. Further we put

$$
M=\max _{x \in \bar{D}, 0 \leqq \varepsilon \leqq \varepsilon_{0}} f_{u}(x, \varepsilon) .
$$

It is easily seen, from the proof of Theorem $2.4 \mathrm{i}$ ), that there exists a positive number $t_{1}$ such that $\phi \in \operatorname{Dom}\left(Q\left(t_{1}\right)\right)$ and that

$$
0 \leqq Q(t) w-v<\varepsilon_{0}, \quad 0 \leqq t \leqq t_{1}
$$

for any continuous function $w$ satisfying $0 \leqq w-v \leqq \varepsilon_{0} / 2$. Because $f(x, Q(t) w)-f(x, v) \leqq M\{Q(t) w-v\}$ so long as $0 \leqq t \leqq t_{1}$, we get

$$
Q\left(t_{1}\right) w-v \leqq e^{M t_{1}} \int_{D} U\left(x, y, t_{1}\right)\{w(y)-v(y)\} d y
$$

by the aid of the comparison theorem (cf. (3.7)). As 


$$
\begin{aligned}
& \left|\nabla_{x} \int_{D} U\left(x, y, t_{1}\right)\{w(y)-v(y)\} d y\right| \\
& \quad \leqq\|w-v\|_{L^{\infty}(D)} \int_{D}\left|\nabla_{x} U\left(x, y, t_{1}\right)\right| d y,
\end{aligned}
$$

and since $Q\left(t_{1}\right) w-v$ vanishes on $\partial D$, it follows that

$$
Q\left(t_{1}\right) w-v \leqq c_{1}\|w-v\|_{L^{\infty}(D)} \rho,
$$

where

$$
c_{1}=e^{M t_{1}} \sup _{x \in \bar{D}} \int_{D}\left|\nabla_{x} U\left(x, y, t_{1}\right)\right| d y .
$$

Let $c$ be a small positive constant such that (3.6) in Lemma 3.8 holds for the present $\phi$ and $t_{1}$ (recall $\psi \in \operatorname{Dom}\left(Q\left(t_{1}\right)\right)$ ). As $v$ is unstable upward, there exists a continuous function $\psi_{1}(x)$ which satisfies the inequalities

$$
0 \leqq \psi_{1}-v \leqq \min \left(\frac{c}{c_{1}}, \frac{\varepsilon_{0}}{2}\right)
$$

on $\bar{D}$ and also the inequality

$$
\left[Q\left(t_{0}\right) \psi_{1}\right]\left(x_{0}\right)-v\left(x_{0}\right) \geqq \varepsilon_{0}
$$

at some $\left(x_{0}, t_{0}\right)$. Here $t_{0}>t_{1}$ because of (3.8). Combining (3.6) and (3. 9), we get

$$
Q\left(t_{1}\right) \psi_{1} \leqq v+c \rho \leqq Q\left(t_{1}\right) \psi,
$$

so it follows from the comparison theorem and (3.10) that

$$
\left[Q\left(t_{0}\right) \psi\right]\left(x_{0}\right)-v\left(x_{0}\right) \geqq \varepsilon_{0},
$$

since $t_{0}>t_{1}$. As $\psi$ is any element of $X^{+}(v)$, the strong upward instability of $v$ is established.

Secondly, the case where (A), (B2) hold. Using Lemma 3.9 instead of Lemma 3.8, we obtain the conclusion more easily. Q.E.D.

\section{§ 4. Hultiple Existence of Equilibrium Solutions}

In this section we consider problems under both of the hypotheses (A) and (B) (see $\S 2$ and $\S 3$ ), so the boundary condition of (1.1) can be expressed in one of the forms 


$$
u=\beta(x),
$$

or

$$
\frac{\partial u}{\partial \nu}+\sigma(x) u=\gamma(x)
$$

Definition 3. A closed set $Y$ in $C^{1}(\bar{D}) \cap C^{2}(D)$ has the property $(S)$ if there exists a family of sets $\left\{Y_{\alpha}\right\}_{\alpha \in A}$ in $C^{1}(\bar{D}) \cap C^{2}(D)$ which satisfies the following conditions:

1) $\bigcap_{\alpha \in A} Y_{\alpha}=Y$;

2) for any $\alpha_{1}, \alpha_{2} \in A$ there exists an $\alpha_{3} \in A$ with $Y_{\alpha_{3}} \subset Y_{\alpha_{1}} \cap Y_{\alpha_{2}}$;

3) each $Y_{\alpha}$ is closed in $C^{1}(\bar{D}) \cap C^{2}(D)$ and bounded in $L^{\infty}(D)$;

4) given any element $w$ of $Y$, any $\alpha \in A$ and any $\delta>0$, there exist continuous functions $w_{1}, w_{2}$ and a finite number $T \geqq 0$ such that

$$
w-\delta \leqq w_{1} \leqq w \leqq w_{2} \leqq w+\delta, \quad w_{1}, w_{2} \neq w,
$$

and that

$$
Q(t) w_{i} \in Y_{\alpha}, \quad i=1,2,
$$

for $T \leqq t \leqq \infty$, where $Q$ is the operator introduced in (2.8).

Remark 4.1. If a closed plus invariant set $Y$ is plus stable, i.e., if given any neighborhood $U$ of $Y$ there exists a neighborhood $V \supset Y$ such that $Q(t) V \subset U$ for $t \geqq 0$, then $Y$ has the property $(S)$.

Now we present our main results in this section, which consist of the following three theorems. The first one may seem rather different from the others because of its abstract form, but one will find the second theorem to be an immediate consequence of it, and in $\S 6$ will be found a more concrete and more interesting application of it.

Theorem 4.2. Let (A), (B) hold and let $Y$ be a nonempty closed set in $C^{1}(\bar{D}) \cap C^{2}(D)$ which has the property $(S)$. Then $Y$ contains at least onc stable solution of (1.2).

Theorem 4. 3. Let (A), (B) hold and let $v_{1}, v_{2}$ be distinct 
solutions of (1.2). Suppose $v_{1} \leqq v_{2}$ on $\bar{D}$ and that $v_{1}$ is unstable upward while $v_{2}$ downward. Then

i) there exist a minimal solution $v_{3}$ and a maximal solution $v_{4}$ in such a sense that

$$
v_{1} \leqq v_{3} \leqq v \leqq v_{4} \leqq v_{2}, \quad v_{1} \not \equiv v_{3}, \quad v_{4} \not \equiv v_{2}
$$

for any solution $v$ satisfying $v_{1} \leqq v \leqq v_{2}, v \not v_{1}, v_{2}$;

ii) there exists at least one stable solution $v$ satisfying $v_{1} \leqq v$ $\leqq v_{2}$.

Theorem 4. 1. Let (A), (B) hold and let $v_{1}, v_{2}$ be distinct solutions of (1.2) satisfying $v_{1} \leqq v_{2}$. Suppose $v_{1}$ is stable from above and $v_{2}$ from below. Then there exists at least one other solution $v$ rehich satisfies $v_{1} \leqq v \leqq v_{2}$.

Here we say $v$ is stable from above (resp. below) if it is not unstable upward (resp. downward). As a result of Theorems 4.3 and 4. 4 we have the following corollary:

Corollary 4. 5. Let (A), (B) hold and lel $v_{1}, v_{2}$ be distinct solutions of (1.2). Suppose that $v_{1} \leqq v_{2}$ and that there exists no other solution lying between $v_{1}$ and $v_{2}$. Then, either

$$
\lim _{t \rightarrow \infty} Q(t) w=v_{1} \quad \text { in } \quad C^{1}(\bar{D}) \cap C^{2}(D)
$$

for all $w \in X^{+}\left(v_{1}\right) \cap X^{-}\left(v_{2}\right)$ or

$$
\lim _{t \rightarrow \infty} Q(t) \tau=v_{2} \quad \text { in } \quad C^{1}(\bar{D}) \cap C^{2}(D)
$$

for all $w \in X^{+}\left(v_{1}^{\prime}\right) \cap X^{-}\left(v_{2}\right)$.

The proof is immediate if Theorem 3.1 i) and Theorem 3.4 are taken into account.

Remark 4.6. 1) One may expect that a similar assertion still holds when $v_{2}$ is replaced by $+\infty$ or $v_{1}$ by $-\infty$. But, so far, any satisfactory result has not been obtained except for the case where $n=1$ 
and the function $f$ is independent of $x$. For this special case we state the result without proof.

Let $n$ and $f$ be just as above and let $v$ be a solution of (1.2) satisfying $S^{+}(v)=\varnothing \quad\left(\right.$ resp. $\left.S^{-}(v)=\varnothing\right)$. Then we have the following alternatives:

$$
\lim _{t \rightarrow \infty} Q(t) w=v \quad \text { in } \quad C^{1}(\bar{D}) \cap C^{2}(D)
$$

for all $w \in X^{+}(v)$ (resp. $X^{-}(v)$ ) or

$$
\begin{aligned}
& \lim _{t \rightarrow T} \max _{x \in \bar{D}}[Q(t) w](x)=+\infty \\
& \text { (resp. } \left.\lim _{t \rightarrow T} \min _{x \in \bar{D}}[Q(t) w](x)=-\infty\right)
\end{aligned}
$$

for all $w \in X^{+}(v)$ (resp. $X^{-}(v)$ ), where $T$ is a positive number or infinity depending on $w$.

2) The same is truc in the case where $n=1$ and $f(x, u)$ is bounded from below (resp. above) in the domain $D \times(0,+\infty)$ (resp. $D \times(-\infty, 0))$. We can prove these results by using Corollary 4.5.

From this remark, we get rather a simple sufficient (and necessary) condition for the instability of a solution. Given such a solution as in the above remark, suppose we could find a continuous function w such that $Q(t)$ w blows up to $-\infty$ (resp. $-\infty$ ) in a finite time or diverges to $+\infty$ (resp. $-\infty$ ) as $t \rightarrow \infty$; then the upward (resp. downward) instability of $v$ follows. In fact, the former alternative in Remark 4.6. 1) cannot hold in this case by virtue of the comparison theorem.

In finding such a function $w$ the idea of upper and lower solutions is often useful. For, if $w$ is a lower (resp. an upper) solution satisfying $w\left(x_{0}\right)>v\left(x_{0}\right)$ (resp. $w\left(x_{0}\right)<v\left(x_{0}\right)$ ) at some $x_{0} \in D$, it is easily verified that $Q(t) w$ is monotone increasing (resp. decreasing) in $t$ to finally blow up or diverge to $+\infty$ (resp. $-\infty$ ) (see [12; Remark in $\S 4]$ ). So the question is merely how to find such a lower or an upper solution. This method can be applied, for example, to the boundary value problem $v^{\prime \prime}+e^{v}=0,\left.v\right|_{\partial D}=0$. In fact, given any positive number $M$, a lower solution $w$ satisfying $w\left(x_{0}\right)>M$ at some $x_{0} \in D$ is always found, and so we can a priori conclude that any solution of this problem above 
which there lies no other solution is unstable upward in the sense that the second alternative in Remark 4.6 1) holds. (Instability of such solutions has already been shown in H. Fujita [6] under the hypothesis that the problem has at least two solutions.)

Now, let us begin the proof of the theorems.

Proof of Theorem 4.2. Suppose $Y$ does not contain any stable solution of (1.2). By definition, there exists a family of sets $\left\{Y_{\alpha}\right\}_{\alpha \in A}$ which satisfies the conditions stated in Definition 3. So, for any $\alpha \in A$, we can find a continuous function $w_{\alpha}$ such that $Q(t) w_{\alpha} \in Y_{\alpha}$ for all $t \geqq T_{\alpha}$, where $T_{\alpha}$ is a finite number depending on $\alpha$ and $w_{\alpha}$. Since $Y_{\alpha}$ is closed in $C^{1}(\bar{D}) \cap C^{2}(D)$ and bounded in $L^{\infty}(D)$, it follows from Theorem 2.8 that $\Omega\left(w_{\alpha}\right)$ is a nonempty subset of $Y_{\alpha}$. Set $K=$ closure $\bigcup_{\alpha \in A} \Omega\left(w_{\alpha}\right)$, where the closure is with respect to $C^{1}(\bar{D}) \cap C^{2}(D) . \quad K$ is a set of solutions of (1.2) and, by Corollary 2.5, $Y_{\alpha} \cap K$ is nonempty and compact. Considering the conditions 1) and 2), we see $\bigcap_{\alpha \in A}\left(Y_{\alpha} \cap K\right)$ $=Y \cap K \neq \varnothing$, which implies that $Y$ contains at least one solution of (1.2). Let $v_{0} \in Y \cap K$. As it is supposed that $Y$ contains no stable solution, $v_{0}$ must be unstable upward or unstable downward. Without loss of generality we can assume the upward instability of it. Then, as is seen in Theorem 3.4, it is strongly unstable upward.

Now let $Z$ be the set of all the solutions of (1.2) that are unstable upward and contained in $Y$. Naturally $Z$ is regarded as a partially ordered set (the relation $v_{1} \leqq v_{2}$ means $v_{1}(x) \leqq v_{2}(x)$ everywhere in $\left.\bar{D}\right)$. Denote by $M$ the set of all the well-ordered subset of $Z$. Again can $M$ be regarded as a partially ordered set with respect to the following order relation: $W_{1} \leqq W_{2}$ if $W_{1}$ coincides with a segment of $W_{2}$. Clearly, $M$ is an inductively ordered set and is not empty as it contains $\left\{v_{0}\right\}$. Therefore, by Zorn's lemma, there exists a maximal element $W$ of $M$. Here we have two cases.

Case 1. Suppose that $W$ has its greatest element $\widetilde{v}$, in other words, consider the case where $W$ is isomorphic to the segment of an isolated ordinal number. In this case the minimum of $S^{+}(\widetilde{\mathcal{V}})$ exists and belongs to $Z$. To see this, we first note that there exists a function $w \in X^{+}(\widetilde{v})$ such that $Q(t) w$ remains bounded in $L^{\infty}(D)$ as $t \rightarrow \infty$ (see Definition 3 
and recall that each $Y_{\alpha}$ is bounded in $\left.L^{\infty}(D)\right)$. So, considering that $\widetilde{v}$ is unstable upward, we find from Theorem 3.1 that $S^{+}(\widetilde{v})$ is not empty and has its minimum $\widetilde{v}^{+}$. Again by definition, given any $\delta>0$ and any $\alpha \in A$, there exists a function $\phi \in X^{+}(\widetilde{v}) \cap X^{-}(\widetilde{v}+\delta)$ satisfying $Q(t) \psi \in Y_{\alpha}$ on some interval $T \leqq t<\infty$. Here, taking a sufficiently small $\delta$, we can make $\widetilde{v} \leqq Q\left(t_{1}\right) \psi \leqq \widetilde{v}^{+}$for some $t_{1} \geqq 0$. (In the case where (B2) holds, $\widetilde{v}^{+} \geqq \widetilde{v}+c$ for some $c>0$ by virtue of Lemma 3.9. So the above statement is trivial if we put $\delta=c$ and $t_{1}=0$. In the case where (B1) holds, $\widetilde{v}^{+} \geqq \widetilde{v}+c \rho$ for some $c>0$ by virtue of Lemma 3. 8. Therefore, as is seen in the proof of Theorem 3.4, we can find such a $\delta>0$ and a $t_{1} \geqq 0$ as above.) It means that $Q(t) \psi$ converges to $\widetilde{v}^{+}$in $C^{1}(\bar{D}) \cap C^{2}(D)$ as $t \rightarrow \infty$ (see Theorem $3.1 \mathrm{i}$ )). Hence $\widetilde{v}^{+}$is contained in $Y_{\alpha}$. As $\alpha$ is any element of $A, \widetilde{v}^{+}$is also contained in $Y$. Seeing that $Q(t) \psi$ converges to $\widetilde{v}^{+}$from below, we find, by the aid of Corollary 3.5, that $\widetilde{v}^{+}$ is stable from below. So it must be unstable upward since $Y$ contains no stable solution. Consequently $\widetilde{v}^{+}$belongs to $Z$ and hence $W \cup\left\{\widetilde{v}^{+}\right\}$ to $M$.

Case 2. Suppose that $W$ does not have the greatest element, in other words, consider the case where $W$ is isomorphic to the segment of a limit ordinal number. In this case, putting

$$
\widehat{v}(x)=\sup _{v \in W} v(x),
$$

we claim that $\widehat{v}$ belongs to $Z$. In fact, since $Y$ is bounded in $L^{\infty}(D)$, $\widehat{v}$ is an accumulation point of $W$ in the topology of $C^{1}(\bar{D}) \cap C^{2}(D)$. So $\widehat{v}$ is a solution of (1.2). The relation $\widehat{v} \in Y$ and the stability of $\widehat{v}$ from below follow from the closedness of $Y$ and Corollary 3.5 respectively. So $\widehat{v}$ belongs to $Z$.

Thus, in either case, the existence of an element that violates the maximality of $W$ has been shown. So our supposition that $Y$ contains no stable solution must be false.

Q.E.D.

Proof of Theorem 4.3. i) As $v_{2} \in S^{+}\left(v_{1}\right), S^{+}\left(v_{1}\right)$ is not empty, and so it follows from Theorems 3.1 and 3.4 that the minimum of $S^{+}\left(v_{1}\right)$ exists. We denote it by $v_{3}$. Not only $v_{1} \leqq v_{3} \leqq v_{2}$ and $v_{1} \not \equiv v_{3}$, but also $v_{3} \not \equiv v_{2}$ since $v_{3}$ is stable from below while $v_{2}$ is not. Considering 
that $v_{3} \in S^{-}\left(v_{2}\right)$, we can similarly show the existence of $v_{4}=\max S^{-}\left(v_{2}\right)$, which satisfies

$$
v_{3} \leqq v_{4} \leqq v_{2}, \quad v_{4} \not \equiv v_{2} .
$$

The rest of i) follows immediately.

ii) Let $v_{3}, v_{4}$ be as above and put

$$
Y=\left\{w \in C^{1}(\bar{D}) \cap C^{2}(D) ; v_{3}-\bar{v} / 4 \leqq w \leqq v_{4}+\overline{\bar{v}} / 4\right\},
$$

where $\bar{v}=v_{3}-v_{1}$ and $\overline{\bar{v}}=v_{2}-v_{4}$. Note that in the case where (A), (B1) (resp. (A), (B2)) hold there exists a constant $c>0$ such that $\bar{v}, \overline{\bar{v}} \geqq c \rho$ (resp. $\geqq c)$ thanks to Lemma 3.8 (resp. 3.9). So there exists a finite number $T \geqq 0$ such that

$$
Q(t)\left(v_{3}-\bar{v} / 2\right), \quad Q(t)\left(v_{4}+\overline{\bar{v}} / 2\right) \in Y
$$

for all $t \geqq T$, because these two functions converge to $v_{3}, v_{4}$, respectively in $C^{1}(\bar{D})$ as $t \rightarrow \infty$. It follows from the comparison theorem that $Q(t) w \in Y$ for all $t \geqq T$ and any function $w \in C^{1}(\bar{D}) \cap C^{2}(D)$ satisfying $v_{3}-\bar{v} / 2 \leqq w \leqq v_{4}+\overline{\bar{v}} / 2$. Putting $Y_{\alpha}=Y$ we easily find that $Y$ has the property $(S)$. The conclusion now follows from Theorem 4.2.

Q.E.D.

Proof of Theorem 4.4. First we prove this theorem under the assumptions (A), (B1). Suppose $X^{+}\left(v_{1}\right) \cap X^{-}\left(v_{2}\right)$ contains no solution of (1.2). Then it follows from the comparison theorem, Theorem 2.8 ii) and Corollary 2.11 that for any $w \in X^{+}\left(v_{1}\right) \cap X^{-}\left(v_{2}\right) Q(t) w$ converges either to $v_{1}$ or to $v_{2}$ as $t \rightarrow \infty$ in $C^{1}(\bar{D}) \cap C^{2}(D)$. Set

$$
w_{\theta}=v_{1}+\theta\left(v_{2}-v_{1}\right)
$$

for $0 \leqq \theta \leqq 1$. By virtue of the comparison theorem there exists a number $\theta_{0}$ such that

$$
\begin{aligned}
& Q(t) w_{\theta} \rightarrow v_{1}(\text { as } t \rightarrow \infty) \text { if } 0 \leqq \theta<\theta_{0}, \\
& Q(t) w_{\theta} \rightarrow v_{2}(\text { as } t \rightarrow \infty) \text { if } \theta_{0}<\theta \leqq 1 .
\end{aligned}
$$

Neither $\theta_{0}=0$ nor $\theta_{0}=1$ because of the stability of $v_{1}$ and $v_{2}$. Without loss of generality we can assume $Q(t) w_{\theta_{0}} \rightarrow v_{1}$ as $t \rightarrow \infty$. Since this convergence occurs in $C^{1}(\bar{D})$, given any $c>0$ there exists a $t^{\prime} \geqq 0$ such 
that

$$
v_{1} \leqq Q\left(t^{\prime}\right) w_{\theta_{0}} \leqq v_{1}+c \rho,
$$

where $\rho(x)=\operatorname{dist}(x, \partial D)$. Considering

$$
v_{2}-v_{1} \geqq c_{0} \rho
$$

holds for some $c_{0}>0$ by virtue of Lemma 3. 8, we then find that

$$
v_{1} \leqq Q\left(t_{1}\right) w_{\theta_{0}} \leqq w_{\theta_{0} / 4}
$$

for some $t_{1}>0$. By the way there exists a constant $c_{1}>0$ such that for any $w \in X^{+}\left(v_{1}\right) \cap X^{-}\left(v_{2}\right)$

$$
\left\|Q\left(t_{1}\right) w_{\theta_{0}}-Q\left(t_{1}\right) w\right\|_{L^{\infty}(D)} \leqq c_{1}\left\|w_{\theta_{0}}-w\right\|_{L^{\infty}(D)} \rho .
$$

This inequality can be shown just similarly to (3.9). Combining (4.2), (4.3) and (4.4), we obtain by a simple calculation the inequality

$$
Q\left(t_{1}\right) w_{\theta} \leqq v_{1}+\left\{\frac{\theta_{0}}{4}+c_{0}^{-1} c_{1}\left(\theta-\theta_{0}\right)\left\|v_{2}-v_{1}\right\|_{L^{\infty}(D)}\right\}\left(v_{2}-v_{1}\right)
$$

for any $\theta$ satisfying $\theta_{0} \leqq \theta \leqq 1$. Let $\theta_{1}$ satisfy

$$
0<\theta_{1}-\theta_{0}<\frac{1}{4} \theta_{0} c_{0} c_{1}^{-1}\left(\left\|v_{2}-v_{1}\right\|_{L^{\infty}(D)}\right)^{-1}
$$

It then follows from (4.5) that $Q\left(t_{1}\right) w_{\theta_{1}} \leqq w_{\theta_{0} / 2}$. So we can see from (4. 1a) that $Q\left(t+t_{1}\right) w_{\theta_{1}}$ converges to $v_{1}$ as $t \rightarrow \infty$. On the other hand, since $\theta_{1}>\theta_{0}, Q(t) w_{\theta_{1}}$ must converge to $v_{2}$ by virtue of $(4.1 \mathrm{~b})$. This contradiction proves the existence of at least one solution of (1.2) between $v_{1}$ and $v_{2}$.

Secondly', under the assumptions (A), (B2). The proof is just similar to the above one, except that we use Lemma 39 instead of Lemma 3.8.

Q.E.D.

\section{Chapter II. Homogeneous Neumann Problems}

\section{$\S 5$. Instability of Non-constant Equilibrium Solutions}

In this section, and also in next section, we study a special form of (1. 1): 


$$
\frac{\partial u}{\partial t}=\Delta u+f(u)
$$

$$
u(x, 0)=u_{0}(x),
$$

$$
\frac{\partial u}{\partial n}=0 \text {. }
$$

The stationary equation corresponding to (5.1) is (1.3). Note that $f$ is assumed to be independent of $x$. So assumptions (A), (B) are put into the reduced form
(A') 1) $D$ is bounded and $\partial D$ is smooth;
2) $f$ is of class $C^{2}$.

Our aim in this section is to pick out a few types of domains in $\boldsymbol{R}^{n}$ in which any stable solution of the boundary value problem (1.3) is necessarily a trivial solution, i.e., a zero of $f(u)$. We mention here three types; convex domains, bodies of rotation with convex sections and domains bounded by concentric spheres. From the standpoint of practial applications, it is important to determine, for a given function $f$, in what domain (1.3) admits non-constant stable solutions and in what domain not. Our results here form part of the answer to the latter question, and part of the answer to the former is given in $\S 6$.

To define the above-mentioned second type of domains exactly, let us introduce the following notation:

$$
G(U)=\left\{U^{-1} T_{\theta} U\right\}_{0 \leqq \theta<2 \pi},
$$

where

$$
T_{\theta}=\left(\begin{array}{rrrr}
\cos \theta & -\sin \theta & & \\
\sin \theta & \cos \theta & & \\
& & 1 & \\
0 & & \ddots & \\
& & &
\end{array}\right)
$$

and $U$ denotes an element of $S O(n)$. Here it is assumed that $n \geqq 2$. We consider $G(U)$ a one-parameter group of transformations. Obviously $G(U)$ is a subgroup of $S O(n)$. 
Definition 4. A subset $Y$ of $\boldsymbol{R}^{n}$ is $G(U)$-invariant if

$$
U^{-1} T_{\theta} U x \in Y
$$

for all $x \in Y$ and $0 \leqq \theta<2 \pi$. A function $w(x)$ is $G(U)$-invariant if its domain is $G(U)$-invariant and

$$
w\left(U^{-1} T_{\theta} U x\right)=w(x)
$$

for all $x, \theta$.

It is easy to see that $Y$ is $G(U)$-invariant if and only if $U Y(=\{U x$; $x \in Y\})$ is $G(E)$-invariant, where $E$ is the unit matrix. When $n=3$, $G(E)$ is nothing but a group of rotations around $x_{3}$-axis.

In a $G(E)$-invariant domain it is convenient to introduce a new coordinate system $\left(\theta, r, x_{3}, \cdots, x_{n}\right)$ instead of the usual orthogonal coordinate system $\left(x_{1}, \cdots, x_{n}\right)$, where

$$
\begin{aligned}
& x_{1}=r \cos \theta, \\
& x_{2}=r \sin \theta,
\end{aligned}
$$

for $r \geqq 0,0 \leqq \theta<2 \pi$. A domain $D$ is $G(E)$-invariant if and only if there exists an $(n-1)$-dimensional domain $D_{0}$ in $\left(r, x_{3}, \cdots, x_{n}\right)$-space such that

$$
D=[0,2 \pi) \times D_{0} .
$$

(Note that for each $x_{3}, \cdots, x_{n}$, the set $\left\{\left(\theta, 0, x_{3}, \cdots, x_{n}\right) ; 0 \leqq \theta<2 \pi\right\}$ is identified to one point.) Similarly, a function $w=w\left(\theta, r, x_{3}, \cdots, x_{n}\right)$ is $G(E)$-invariant if and only if $w$ is independent of $\theta$.

Definition 5. Let $D$ be a $G(E)$-invariant domain. The E-section of $D$ is a domain in $\left(r, x_{3}, \cdots, x_{n}\right)$-space defined by $D_{0}$ in (5.2). We denote it by $S_{E}(D)$. For any $U \in S O(n), U$-section of a $G(U)$-invariant domain $D$ is defined by $S_{U}(D)=S_{E}(U D)$.

Now we are ready to form a clear definition of the three types of domains.

(T1): $D$ is convex.

(T2): $D$ is $G(U)$-invariant for some $U \in S O(n), \overline{U D}$ does not meet 
with the 2-codimensional subspace $x_{1}=x_{2}=0$ (i.e. $r=0$ ) and $S_{U}(D)$ is convex. (Here $n \geqq 2$.)

(T3): $D=\left\{x \in \boldsymbol{R}^{n} ; r_{1}<|x|<r_{2}\right\}$ for some $0<r_{1}<r_{2} . \quad(n \geqq 2$.)

These conditions are incompatible with each other, except that (T2) and (T3) are equivalent conditions when $n=2$. A typical example of domain of type (T2) for $n=3$ is the inside of a torus.

Theorem 5.1. Let ( $\left.\mathbf{A}^{\prime}\right)$ hold, let $D$ be of one of the types (T1) $\sim($ T3) and let $v$ be a solution of (1.3). Suppose $v$ is not a constant function. Then it is unstable in the following sense:

i) If $A^{+} \equiv\left\{\alpha \in \boldsymbol{R} ; f(\alpha)=0, \alpha \geqq \max _{x \in \bar{D}} v(x)\right\}$ is not empty, then any continuous function $\psi$ satisfying $v \leqq \psi \leqq \alpha^{+}\left(=\min A^{+}\right), \psi \equiv v$ belongs to $\operatorname{Dom}(Q(\infty))$ and

$$
\lim _{t \rightarrow \infty} Q(t) \psi=\alpha^{+}
$$

in the topology of $C^{1}(\bar{D}) \cap C^{2}(D)$, where $Q$ is the operator introduced in (2.8).

ii) If $A^{+}=\varnothing$, then given any continuous function $\psi$ satisfying $\psi \geqq v$ and $\psi \neq \equiv$ there exists a $T \in(0, \infty]$ such that

$$
\lim _{t \rightarrow T} \max _{x \in \bar{D}} Q(t) \psi=+\infty \text {. }
$$

i)', ii)' similar propositions hold good for $A^{-}=\{\alpha \in R ; f(\alpha)=0$, $\left.\alpha \leqq \min _{x \in \bar{D}} v(x)\right\}$

This is our main theorem in Chapter II. The following theorem, though not so strong a statement as above, is also interesting.

Theorem 5. 2. Let ( $\left.\mathbf{A}^{\prime}\right)$ hold, let $D$ be $G(U)$-invariant for some $U \in S O(n)$ and let $v$ be a solution of (1.3). If $v$ is not $G(U)$-invariant, it is unstable both upward and downward.

To see the above results by the use of Theorems 3.1 and 3.3 , we have to show the negativity of the first (i.e., least) eigenvalue $\lambda_{1}$ of the following eigenvalue problem: 


$$
\left\{\begin{array}{l}
\widetilde{L} \varphi+\lambda \varphi=0 \text { in } D, \\
\frac{\partial \varphi}{\partial n}=0 \text { on } \partial D,
\end{array}\right.
$$

where

$$
\widetilde{L} \varphi=\Delta \varphi+f^{\prime}(v(x)) \varphi
$$

As is well-known,

$$
\lambda_{1}=\min _{\varphi \in H^{1}(D),\|\varphi\|_{L^{2}(D)}=1} \mathcal{H}(\varphi)
$$

where

$$
\mathcal{H}(\varphi) \equiv \int_{D}\left\{|\nabla \varphi|^{2}-f^{\prime}(v(x)) \varphi^{2}\right\} d x
$$

and the eigenfunctions corresponding to $\lambda_{1}$ are characterized as to minimize the form

$$
\mathcal{H}(\varphi) /\|\varphi\|_{L^{2}(D)} .
$$

As a consequence of Krein's theorem, $\lambda_{1}$ is a simple eigenvalue and any eigenfunction subject to $\lambda_{1}$ does not change sign in $D$. Therefore, it is sufficient for concluding $\lambda_{1}<0$ if we find such a function $\varphi \in H^{1}(D)$ as to make $\mathscr{H}(\varphi)<0$ or as to make $\mathscr{H}(\varphi)=0$ and change sign in $D$.

Next lemma will play an important role in the sequel.

Lemma 5. 3. Let $D_{1}$ be a convex domain in $\boldsymbol{R}^{n}$ with a smooth boundary'. Suppose w belongs to $C^{3}\left(\bar{D}_{1}\right)$ and satisfies $\partial w / \partial n=0$ on $\partial D_{1}$. Then

$$
\frac{\partial}{\partial n}|\nabla r|^{2} \leqq 0 \quad \text { on } \quad \partial D_{1}
$$

Proof. Let $\rho$ be a function of class $C^{2}$ defined in a neighborhood of $\bar{D}_{1}$ such that

$$
\begin{cases}\rho<0 & \text { in } D_{1} \\ \rho=0 & \text { on } \partial D_{1} \\ \rho>0 & \text { outside } \bar{D}_{1},\end{cases}
$$

and that $\nabla \rho$ never vanishes on $\partial D_{1}$. Since $\nabla \rho$ is outer normal to $\partial D_{1}$ 
at each point of $\partial D_{1}$, we have

$$
\frac{\partial w}{\partial n}=\left.\frac{1}{|\nabla \rho|} \nabla \rho \cdot \nabla w\right|_{\partial D_{1}},
$$

where - denotes the usual Euclidean inner product. So it follows from the boundary condition $\partial w / \partial n=0$ that there exists a function $g \in C^{1}\left(\bar{D}_{1}\right)$ such that

$$
\nabla \rho \cdot \nabla w=\rho g \text { on } \bar{D}_{1} .
$$

Differentiating (5.5) by $x_{i}$ yields

$$
\nabla \rho \cdot \nabla\left(\frac{\partial w}{\partial x_{i}}\right)+\nabla\left(\frac{\partial \rho}{\partial x_{i}}\right) \cdot \nabla w=\frac{\partial \rho}{\partial x_{i}} g+\rho \frac{\partial g}{\partial x_{i}},
$$

for $i=1, \cdots, n$. Multiplying this equality by $\partial w / \partial x_{i}$ and then summing over $i=1, \cdots, n$, we get

$$
\begin{aligned}
\frac{1}{2} \nabla \rho \cdot \nabla & \left(|\nabla w|^{2}\right)+\sum_{i, j} \frac{\partial^{2} \rho}{\partial x_{i} \partial x_{j}} \frac{\partial w}{\partial x_{i}} \frac{\partial w}{\partial x_{j}} \\
& =g \nabla \rho \cdot \nabla w+\rho \nabla g \cdot \nabla w=\rho g^{2}+\rho \nabla g \cdot \nabla w .
\end{aligned}
$$

Therefore

$$
\frac{1}{2} \nabla \rho \cdot \nabla\left(|\nabla w|^{2}\right)+\sum_{i, j} \frac{\partial^{2} \rho}{\partial x_{i} \partial x_{j}} \frac{\partial w}{\partial x_{i}} \frac{\partial w}{\partial x_{j}}=0 \quad \text { on } \quad \partial D_{1},
$$

hence

$$
\frac{\partial}{\partial n}|\nabla w|^{2}=-\frac{2}{|\nabla \rho|} \sum_{i, j} \frac{\partial^{2} \rho}{\partial x_{i} \partial x_{j}} \frac{\partial w}{\partial x_{i}} \frac{\partial w}{\partial x_{j}} \quad \text { on } \partial D_{1}
$$

As is well-known, for each $\tilde{x} \in \partial D_{1}$ the Hessian of $\rho$ at $\tilde{x}$ induces a positive semi-definite quadratic form on the tangent hyperplane to $\partial D_{1}$ at $\widetilde{x}$, because $D_{1}$ is convex. Since the boundary condition $\partial w / \partial n=0$ implies that the vector $\nabla w(\widetilde{x})$ belongs to this tangent hyperplane, it follows that the right-hand side of $(5.6)$ is nonpositive. Q.E.D.

Lemma 5. 4. Let ( $\left.\mathbf{A}^{\prime}\right)$ hold and let $v$ be a solution of (1.3). Suppose $v$ is not a constant and that

$$
\sum_{i=1}^{n} \mathcal{H}\left(\frac{\partial v}{\partial x_{i}}\right) \leqq 0
$$


Then $\lambda_{1}<0$.

Proof. The statement is trivial if $\sum_{i} \mathcal{H}\left(\partial v / \partial x_{i}\right)<0$, for in this case there exists at least one integer $k, 1 \leqq k \leqq n$, such that $\mathscr{H}\left(\partial v / \partial x_{k}\right)$ $<0$. So we assume $\sum_{i} \mathcal{H}\left(\partial v / \partial x_{i}\right)=0$.

Now suppose that $\lambda_{1} \geqq 0$. Then it follows from the above equality that

$$
\mathcal{H}\left(\partial v / \partial x_{i}\right)=0, \quad i=1, \cdots, n,
$$

and that $\lambda_{1}=0$. This means each $\partial v / \partial x_{i}$ is an eigenfunction subject to $\lambda_{1}$ or is identically zero. Since $\lambda_{1}$ is simple, we can find a function $\varphi_{1}(x)$ and constants $c_{i}(i=1, \cdots, n)$ such that

$$
\frac{\partial v}{\partial x_{i}}=c_{i} \varphi_{1}, \quad i=1, \cdots, n
$$

It follows that there exists a function $\Phi$ of one variable satisfying

$$
v\left(x_{1}, \cdots, x_{n}\right)=\Phi\left(c_{1} x_{1}+\cdots+c_{n} x_{n}\right)
$$

for any $\left(x_{1}, \cdots, x_{n}\right) \in \bar{D}$. Here $c \equiv \sqrt{c_{1}^{2}+\cdots+c_{n}^{2}} \neq 0$, because $v$ is not a constant. Put

$$
\begin{aligned}
& a=\min _{x \in \bar{D}}\left(c_{1} x_{1}+\cdots+c_{n} x_{n}\right), \\
& b=\max _{x \in \bar{D}}\left(c_{1} x_{1}+\cdots+c_{n} x_{n}\right)
\end{aligned}
$$

and let $\widetilde{x}$ be the point of $\partial D$ where the above minimum $a$ is attained. It is easy to see that the vector $\left(c_{1}, \cdots, c_{n}\right)$ is inner normal to $\partial D$ at $\widetilde{x}$. Combining (5.7) and (1.3), and considering the above-mentioned fact, we get an ordinary differential equation of the form

$$
\Phi^{\prime \prime}(y)+c^{-2} f(\Phi(y))=0, \quad a<y<b,
$$

with the initial condition

$$
\Phi^{\prime}(a)=0
$$

Recall that each $\partial v / \partial x_{i}(i=1, \cdots, n)$ is an eigenfunction of (5.3) or is identically zero. So it satisfies the Neumann boundary condition on $\partial D$. Consequently,

$$
c_{i} \Phi^{\prime \prime}(a)=0, \quad i=1, \cdots, n
$$


hence $\Phi^{\prime \prime}(a)=0$. It then follows from (5.8a) that

$$
f(\Phi(a))=0 .
$$

Combining (5.8) and (5.9), we find, by the aid of the uniqueness theorem for solutions of ordinary differential equations, that

$$
\Phi(y)=\Phi(a), \quad a \leqq y \leqq b .
$$

It implies that $v$ is a constant, which contradicts the presumption. Therefore our supposition that $\lambda_{1} \geqq 0$ must be false, thus the negativity of $\lambda_{1}$ is established.

Q.E.D.

To consider (1.3) and (5.1) in a $G(E)$-invariant domain, it is convenient to express $\Delta \equiv \sum_{i} \partial^{2} / \partial x_{i}^{2}$ in the coordinates $\theta, r, x_{3}, \cdots, x_{n}$ :

$$
\Delta=\tilde{\Delta}+\frac{1}{r} \frac{\partial}{\partial r}+\frac{1}{r^{2}} \frac{\partial^{2}}{\partial \theta^{2}}, \quad r>0,
$$

where

$$
\tilde{\Delta}=\frac{\partial^{2}}{\partial r^{2}}+\sum_{i=3}^{n} \frac{\partial^{2}}{\partial x_{i}^{2}}
$$

We also use the following formula:

$$
\nabla w_{1} \cdot \nabla w_{2}=\tilde{\nabla} w_{1} \cdot \tilde{\nabla} w_{2}+\frac{1}{r^{2}} \frac{\partial w_{1}}{\partial \theta} \frac{\partial w_{2}}{\partial \theta}, \quad r>0,
$$

where

$$
\tilde{\nabla}=\left(\frac{\partial}{\partial r},-\frac{\partial}{\partial x_{3}}, \cdots, \frac{\partial}{\partial x_{n}}\right) .
$$

It is easily seen from (5.10) that $\Delta$ and $\partial / \partial \theta$ are commutative. Consequently, differentiating (1.3a) by $\theta$ yields

$$
\Delta\left(\frac{\partial v}{\partial \theta}\right)+f^{\prime}(v) \frac{\partial v}{\partial \theta}=0
$$

This equality should be understood to hold as long as $r>0$, since (5.10) is valid for $r>0$. However, putting $\partial / \partial \theta=0$ when $r=0$ and then regarding $\partial / \partial \theta$ as a smooth vector field defined everywhere, we can see (5.12) hold everywhere in $D$. 
Lemma 5. 5. Let $D_{1}$ be a $G(E)$-invariant domain with a smooth boundary and let $w$ be a function of class $C^{3}\left(\bar{D}_{1}\right)$ satisfying the boundary condition $\partial w / \partial n=0$ on $\partial D_{1}$. Then

$$
\frac{\partial}{\partial n}\left(\frac{\partial w}{\partial \theta}\right)=0 \quad \text { on } \quad \partial D_{1}
$$

(As is mentioned above, we understand $\partial w / \partial \theta=0$ if $r=0$.)

Proof. Let $\rho$ be a $G(E)$-invariant function of class $C^{2}$ satisfying (5. 4). (It is not difficult to see that we can actually take such a function 0 .) Combining (5.5) and (5.11). We get

$$
\rho g=\tilde{\nabla} \rho \cdot \tilde{\nabla}_{w},
$$

where $g$ is a function of $C^{1}\left(\bar{D}_{1}\right)$. Differentiating this equality by $\theta$, we then obtain

$$
\rho \frac{\partial g}{\partial \theta}=\tilde{\nabla} \rho \cdot \tilde{\nabla}\left(\frac{\partial w}{\partial \theta}\right)=\nabla \rho \cdot \nabla\left(\frac{\partial w}{\partial \theta}\right) .
$$

So $\partial(\partial w / \partial \theta) / \partial n$ vanishes on $\partial D_{1}$.

Q.E.D.

Proof of Theorem 5.2. First of all, note that if $v$ is a solution of (1.3) then it belongs to $C^{3}(\bar{D})$ because of the smoothness of $f$. It should also be noted that we have only to prove this theorem in the case $U=E$ since $U D$ is $G(E)$-invariant and (1.3) does not change its form under any orthogonal transformation of coordinates.

Using (5.12) and Green's formula, we get

$$
\begin{aligned}
\mathcal{H}\left(\frac{\partial v}{\partial \theta}\right) & \equiv \int_{D}\left\{\left|\nabla\left(\frac{\partial v}{\partial \theta}\right)\right|^{2}-f^{\prime}(v)\left(\frac{\partial v}{\partial \theta}\right)^{2}\right\} d x \\
& =\int_{D}\left\{\left.\nabla\left(\frac{\partial v}{\partial \theta}\right)\right|^{2}+\Delta\left(\frac{\partial v}{\partial \theta}\right) \frac{\partial v}{\partial \theta}\right\} d x \\
& =\int_{\partial D} \frac{\partial v}{\partial \theta} \frac{\partial}{\partial n}\left(\frac{\partial v}{\partial \theta}\right) d \widetilde{x} .
\end{aligned}
$$

Consequently, by Lemma 5. 5 ,

$$
\mathscr{H}\left(\frac{\partial v}{\partial \theta}\right)=0 .
$$


As $v$ is not $G(E)$-invariant, $\partial v / \partial \theta$ is not identically zero. So (5.13) implies that $\lambda_{1} \leqq 0$. If $\lambda_{1}=0$, it follows from (5.13) that $\partial v / \partial \theta$ is an eigenfunction of (5.3) subject to $\lambda_{1}$, hence $\partial v / \partial \theta$ does not change sign in $D$. But this is impossible because

$$
\int_{0}^{2 \pi} \frac{\partial v}{\partial \theta}\left(\theta, r, x_{3}, \cdots, x_{n}\right) d \theta=0 .
$$

Therefore $\lambda_{1}$ must be negative. On applying Theorem 3.3 we get to the completion of the proof.

Q.E.D.

Proof of Theorem 5.1. We first show the negativity of $\lambda_{1}$.

Case (T1): By Green's formula,

$$
\begin{aligned}
\sum_{i=1}^{n} \mathcal{H}\left(\frac{\partial v}{\partial x_{i}}\right) & \equiv \sum_{i} \int_{D}\left\{\left|\nabla\left(\frac{\partial v}{\partial x_{i}}\right)\right|^{2}-f^{\prime}(v)\left(\frac{\partial v}{\partial x_{i}}\right)^{2}\right\} d x \\
& =\sum_{i} \int_{D}\left\{\left|\nabla\left(\frac{\partial v}{\partial x_{i}}\right)\right|^{2}+\Delta\left(\frac{\partial v}{\partial x_{i}}\right) \frac{\partial v}{\partial x_{i}}\right\} d x \\
& =\sum_{i} \int_{\partial D} \frac{\partial v}{\partial x_{i}} \frac{\partial}{\partial n}\left(\frac{\partial v}{\partial x_{i}}\right) d \widetilde{x} \\
& =\frac{1}{2} \int_{\partial D} \frac{\partial}{\partial n}|\nabla v|^{2} d \widetilde{x} .
\end{aligned}
$$

Considering that $v \in C^{3}(\bar{D})$ and applying Lemmas 5.3 and 5.4, we get $\lambda_{1}<0$.

Case (T2): As is mentioned in the proof of Lemma 5.5, we can assume $U=E$. Further we assume that $v$ is $G(E)$-invariant, for otherwise the negativity of $\lambda_{1}$ is already shown in the proof of Theorem 5.2.

Let $\rho$ be as in the proof of Lemma 5.5. Then

$$
\begin{aligned}
\frac{\partial}{\partial n}|\nabla v|^{2} & =\frac{1}{|\nabla \rho|} \nabla \rho \cdot \nabla\left(|\nabla v|^{2}\right) \\
& =\frac{1}{|\tilde{\nabla} \rho|} \tilde{\nabla} \rho \cdot \tilde{\nabla}\left(|\tilde{\nabla} v|^{2}\right) .
\end{aligned}
$$

Here we have used the fact that $|\tilde{\nabla} v|=|\nabla v|$ and that $\partial \rho / \partial \theta=0$. The former follows from the assumption that $\partial v / \partial \theta=0$. Since $S_{E}(D)$ is convex and $\partial D=[0,2 \pi) \times \partial S_{E}(D)$, applying Lemma 5.3 to $D_{1}=S_{E}(D)$ yields the nonpositivity of the right-hand side of (5.15). Combining this 
fact and (5.14), and then applying Lemma 5. 4, we find $\lambda_{1}<0$.

Case (T3): Since $D$ is $G(U)$-invariant for any $U \in S O(n)$, we can assume that $v$ is $G(U)$-invariant for any $U$, for otherwise the statement is already established in the proof of Theorem 5.2. In this case, as is easy to see, $v$ is a function of $|x|$. So we put $v(x)=\Phi(|x|)$. Then

$$
|\nabla v(x)|=\left|\Phi^{\prime}(|x|)\right||\nabla| x||=\left|\Phi^{\prime}(|x|)\right|,
$$

since $|x| \neq 0$ as long as $x \in D$. Hence on the boundary $|x|=r_{i}(i=1,2)$ we have

$$
\begin{aligned}
\frac{\partial}{\partial n}|\nabla v(x)|^{2} & =\frac{\partial}{\partial n}\left\{\Phi^{\prime}(|x|)\right\}^{2} \\
& =(-1)^{i} 2 \Phi^{\prime}(|x|) \Phi^{\prime \prime}(|x|) .
\end{aligned}
$$

Considering that

$$
\Phi^{\prime}(|x|)=(-1)^{i} \frac{\partial v}{\partial n}=0, \quad|x|=r_{i}
$$

for $i=1$, 2, we find, from (5.16), that

$$
\frac{\partial}{\partial n}|\nabla v|^{2}=0 \quad \text { on } \quad \partial D
$$

Combining (5.17), (5.14) and Lemma 5.4, we thus get $\lambda_{1}<0$.

Now that $\lambda_{1}$ is shown to be negative in each case, the rest of the proof is not difficult. For instance we prove i) and ii). Suppose $S^{+}(v)$ $\neq \varnothing$. Since $v$ is strongly unstable upward by virtue of Theorem 3. 3, it follows from Theorem 3.1 that $S^{+}(v)$ has its minimum $v^{+}$. Here, by Cororally 3.5, $v^{+}$is stable from below. And so it is a constant function, for, as is just shown above, any non-constant solution of (1.3) is in these cases unstable both upward and downward. Consequently $v^{+}$ is a zero of $f(u)$, and from the minimality of $v^{+}$in $S^{+}(v)$ follows $v^{+}=\alpha^{+}$. This means that $S^{+}(v) \neq \varnothing$ and $A^{+} \neq \varnothing$ are equivalent, hence the assertions i), ii) follow immediately from Theorem 3.1 i), ii), respectively.

Q.E.D.

\section{§. Domains Admitting Non-constant Stable Solutions}

As is seen in Theorem 5.1 for case (T1), any non-constant solution 
of (1.3) is unstable when $n=1$. But it must be noted that when $n \geqq 2$ (1.3) sometimes has non-constant stable solutions. To show this, we present sufficient conditions on $D$ and $f$ for the existence of such solutions, by which various examples can easily be made.

Let the nonlinear term $f$ be of the form

$$
f(u)=k g(u) \text {, }
$$

where $k$ is a positive constant and $g$ is a function of class $C^{2}$ satisfying

$$
g(a)=g(0)=g(b)=0
$$

for some $a<0<b$ and

$$
\begin{array}{ll}
u \leqq g(u)<0, & a<u<0 \\
0<g(u) \leqq u, & 0<u<b .
\end{array}
$$

From (6.1b) and (6.1c) it follows that $G(u) \equiv \int_{0}^{u} g(w) d w$ is nonnegative in $a \leqq u \leqq b$ and takes its maximum at $u=a$ or at $u=b$. Without loss of generality we can assume that $G(b)$ is the maximum.

Secondly, let $D$ be a bounded domain in $\boldsymbol{R}^{n}$ with a sufficiently smooth boundary and let $D_{1}, D_{2}$ be subdomains of $D$ in each of which Poincaré's second inequality holds, i.e., there exist positive constants $\lambda_{2}\left(D_{1}\right)$ and $\lambda_{2}\left(D_{2}\right)$ such that for any function $w \in H^{1}\left(D_{i}\right)$ the following inequality holds:

$$
\lambda_{2}\left(D_{i}\right)^{-1} \int_{D_{i}}|\nabla w|^{2} d x+\frac{\left(\int_{D_{i}} w d x\right)^{2}}{\int_{D_{i}} d x} \geqq \int_{D_{i}} w^{2} d x \quad(i=1,2) .
$$

(Here these constants are assumed to be the best possible ones. So they are nothing but the second eisenvalues of $-\Delta$ under the Neumann boundary conditions if $\partial D_{i}(i=1,2)$ are smooth.) We need some more notation: Let $\mu$ be the Lebesgue measure in $\boldsymbol{R}^{n}$ and let $J(u)$ be the energy form

$$
J(u)=\int_{D}\left\{\frac{1}{2}|\nabla u|^{2}-F(u)\right\} d x,
$$

where $F(u)=k G(u)$. Finally, put

$$
\begin{array}{r}
R[-,+]=\left\{w \in C^{1}(\bar{D}) \cap C^{2}(D) ; a \leqq w \leqq b \text { on } \bar{D},\right. \\
\left.\qquad \int_{D_{1}} w(x) d x<0, \int_{D_{2}} w(x) d x>0\right\} .
\end{array}
$$


We are now going to show that under suitable conditions (1.3) has stable solutions belonging to $R[-,+]$. Of course any function belonging this set is not a constant function. The essential part of the result in this section is based on Theorem 4.2. To apply this theorem, we must find a subset $Y$ of $R[-,+]$ having the property $(S)$ given in Definition 3. Let us begin with the following lemma:

Lemma 6. 1. Let $f, D, D_{1}$ and $D_{2}$ be as above and put $\varepsilon_{0}=G(b) \min \left\{\mu\left(D_{1}\right) \min \left\{k, \lambda_{2}\left(D_{1}\right)\right\}, \quad \mu\left(D_{2}\right) \min \left\{k, \lambda_{2}\left(D_{2}\right)\right\}\right\}$.

Suppose a function $w \in R[-,+]$ satisfies the inequality

$$
J(w)<\varepsilon_{0}-k G(b) \mu(D) .
$$

Then, $Q(t)$ w belongs to $R[-,+]$ for all $t \geqq 0$.

Proof. Since (6.1a) implies that both $v=a$ and $v=b$ are solutions of (1.3), it follows from the comparison theorem that $a \leqq Q(t) w \leqq b$ for all $t \geqq 0$. So what must be proved are the inequalities

$$
\begin{array}{ll}
\int_{D_{1}} Q(t) w d x<0, & t \geqq 0, \\
\int_{D_{2}} Q(t) w d x>0, & t \geqq 0 .
\end{array}
$$

Suppose, for instance, that (6.3a) does not hold. Then, because of the continuity of $Q(t) w$ in $t$, there exists a positive number $t_{1}$ such that

$$
\int_{D_{1}} Q\left(t_{1}\right) w d x=0
$$

Therefore, seeing (6.2), we get

$$
\begin{aligned}
\int_{D_{1}}\left|\nabla w_{1}\right|^{2} d x & \geqq \lambda_{2}\left(D_{1}\right) \int_{D_{1}} w_{1}^{2} d x \\
& \geqq 2 \lambda_{2}\left(D_{1}\right) \int_{D_{1}} G\left(w_{1}\right) d x,
\end{aligned}
$$

where, for simplicity, the function $Q\left(t_{1}\right) w$ is denoted by $w_{1}$. The latter inequality follows from (6.1b) and (6.1c). On the other hand, by virtue of Lemma 2.7, $J(\underline{Q}(t) w)$ is monotone decreasing with respect to 
$t$ in the interval $(0, \infty)$. As a matter of fact, this monotonicity still holds in $[0, \infty)$, since $w$ belongs to $C^{1}(\bar{D})$ (note that $w$ is the limit of a sequence of functions satisfying the Neumann boundary conditions in the topology of $\left.H^{1}(D)\right)$. Consequently

$$
\begin{aligned}
J\left(w_{1}\right) & \leqq J(w) \\
& <\varepsilon_{0}-k G(b) \mu(D) .
\end{aligned}
$$

So, considering that the inequality

$$
\int_{D \backslash D_{1}}\left\{\frac{1}{2}\left|\nabla w_{1}\right|^{2}-F\left(w_{1}\right)\right\} d x \geqq-k G(b) \mu\left(D \backslash D_{1}\right)
$$

holds because of the maximality of $G(b)$, we find

$$
\int_{D_{1}}\left\{\frac{1}{2}\left|\nabla w_{1}\right|^{2}-F\left(w_{1}\right)\right\} d x<\varepsilon_{0}-k G(b) \mu\left(D_{1}\right) .
$$

It follows from (6.4) and (6.5) that

$$
\left\{\lambda_{2}\left(D_{1}\right)-k\right\} \int_{D_{1}} G\left(w_{1}\right) d x<\varepsilon_{0}-k G(b) \mu\left(D_{1}\right) .
$$

Hence, using the inequalities

$$
0 \leqq \int_{D_{1}} G\left(w_{1}\right) d x \leqq G(b) \mu\left(D_{1}\right)
$$

we obtain

$$
\begin{aligned}
\varepsilon_{0} & >\left\{\lambda_{2}\left(D_{1}\right)-k\right\} \int_{D_{1}} G\left(w_{1}\right) d x+k G(b) \mu\left(D_{1}\right) \\
& \geqq G(b) \mu\left(D_{1}\right) \min \left\{k, \lambda_{2}\left(D_{1}\right)\right\},
\end{aligned}
$$

which, however, is impossible by the definition of $\varepsilon_{0}$. This contradiction proves (6.3a). Similarly (6.3b) can be verified.

Q.E.D.

Now we are ready to present the main theorem in this section.

Theorem 6.2. Let $f, D, D_{1}$ and $D_{2}$ be as above, and suppose that there exists a function $w_{0} \in R[-,+]$ satisfying

$$
J\left(w_{0}\right)<\varepsilon_{0}-k G(b) \mu(D),
$$

where $\varepsilon_{0}$ is the number introduced in Lemma 6.1. Then, (1.3) 
has at least one stable solution belonging to $R[-,+]$.

Proof. Let $J_{0}$ be the right-hand side of (6.6) and set

$$
Y_{m}=\left\{w \in R[-,+] ; J(w) \leqq \frac{m J\left(w_{0}\right)+J_{0}}{m+1}\right\}
$$

for $m=1,2, \cdots$. It is clear from Lemma 6.1 and from the monotone decreasing property of $J(Q(t) w)$ that each $Y_{m}$ is invariant under $Q(t)$, i.e.,

$$
Q(t) w \in Y_{m}, \quad t \geqq 0
$$

for all $w \in Y_{m}$. Moreover, each $Y_{m}$ is bounded in $L^{\infty}(D)$ and closed in $C^{1}(\bar{D}) \cap C^{2}(D)$. So it is not difficult to see that $Y=\bigcap_{m=1}^{\infty} Y_{m}$ has the property $(S)$, for $Y$ is contained in the interior of each $Y_{m}$ in the topology of $C^{1}(\bar{D}) \cap C^{2}(D)$. The nonemptiness of $Y$ follows from the fact that $w_{0} \in Y$. Therefore, by Theorem 4.2, $Y$ contains at least one stable solution of (1.3). Considering $Y \subset R[-,+]$, we get to the completion of the proof.

Q.E.D.

It may not be immediately clear that the sufficient conditions given in the above theorem are really possible conditions. So we next make il concrete description of a domain with which above conditions can be realized.

(P) 1) $D$ is a bounded domain in $\boldsymbol{R}^{n}$ with a smooth boundary.

2) $D_{1}$ and $D_{2}$ are subdomains of $D$ in each of which Poincaré's second inequality holds (such as convex domains, domains with piecewise smooth boundaries, and the like).

3) There exists a connected component (or a union of connected components) of $D \cap\left\{x ;-\frac{l}{2} \leqq x_{1} \leqq \frac{l}{2}\right\}$, denoted by $D_{3}$, such that $D \backslash D_{3}$ is divided into disjoint open sets $O_{1}$ and $O_{2}$ containing $D_{1}$ and $D_{2}$ respectively and satisfying $\partial D_{3} \cap \partial O_{1} \subset\left\{x ; x_{1}=-\frac{l}{2}\right\}$ and $\partial D_{3} \cap \partial O_{2} \subset\left\{x ; x_{1}=\frac{l}{2}\right\}$ (see Figure).

4) The $(n-1)$-dimensional measure of the intersection of $D_{3}$ and the hyperplane $\left\{x ; x_{1}=\xi\right\}$ does not exceed $S$ for $-\frac{l}{2} \leqq \xi \leqq \frac{l}{2}$. 


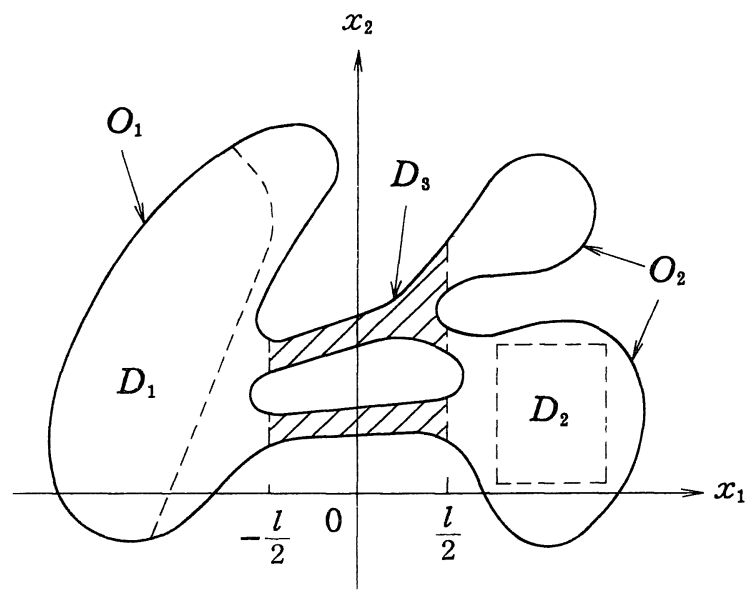

Figure Example of domain $D$ for $n=2$.

In terms of the above notation we can derive from Theorem 6.2 some more simplified sufficient conditions:

Corollary 6.3. Let (P) hold and let $f(u)=k g(u)$ satisfy (6. 1). Suppose

$$
\left\{\frac{(b-a)^{2}}{2 l}+k G(b) l\right\} S \leqq \varepsilon_{0}-k\{G(b)-G(a)\} \mu\left(O_{1}\right),
$$

where $\varepsilon_{0}$ is as in Lemma 6.1. Then (1.3) has at least one stable solution belonging to $R[-,+]$.

Proof. To apply the previous theorem, we must show the existence of such a function $w_{0} \in R[-,+]$ as to satisfy (6.6). Let $\widetilde{w}$ be a function on $D$ such that

$$
\widetilde{w}= \begin{cases}a & \left(x \in O_{1}\right) \\ \frac{a+b}{2}+\frac{(b-a) x_{1}}{l} & \left(x \in D_{3}\right) \\ b & \left(x \in O_{2}\right) .\end{cases}
$$

Obviously $\widetilde{w}$ belongs to $H^{1}(D)$ and satisfies

$$
\int_{D_{1}} \widetilde{w}(x) d x<0, \quad \int_{D_{2}} \widetilde{w}(x) d x>0 .
$$


In addition,

$$
\begin{aligned}
J(\widetilde{\boldsymbol{w}})= & -k G(a) \mu\left(O_{1}\right)-k G(b)\left\{\mu\left(D_{3}\right)+\mu\left(O_{2}\right)\right\} \\
& +\int_{D_{3}}\left\{\frac{1}{2}|\nabla \widetilde{\widetilde{w}}|^{2}-k G(\widetilde{\boldsymbol{w}})+k G(b)\right\} d x \\
< & -k G(a) \mu\left(O_{1}\right)-k G(b)\left\{\mu\left(D_{3}\right)+\mu\left(O_{2}\right)\right\} \\
& +\frac{(b-a)^{2}}{2 l} S+k G(b) l S \\
\leqq & \varepsilon_{0}-k G(b) \mu(D) .
\end{aligned}
$$

Therefore, as $C^{1}(\bar{D}) \cap C^{2}(D)$ is dense in $H^{1}(D)$, there exists a function $w_{0} \in R[-,+]$ satisfying (6.6). Hence, by Theorem 6.2, $R[-,+]$ contains at least one stable solution of (1.3). Q.E.D.

Remark 6. 4. From the above corollary it immediately follows that given any function $f$ satisfying (6.1) there exists a domain $D$ such that (1.3) has a non-constant stable solution. To see this, put $\mu\left(D_{1}\right)=\mu\left(O_{1}\right)$ $\leqq \mu\left(D_{2}\right)$ and $k \leqq \lambda_{2}\left(D_{i}\right) \quad(i=1,2)$, and then take a sufficiently small $S$.

\section{References}

[1] Amann, H., On the existence of positive solutions of nonlinear elliptic boundary value problems, Indiana Univ. Math. J., 21 (1971), 125-146.

[2] - Existence of multiple solutions for nonlinear elliptic boundary value problems, Indiana Univ. Math. J., 21 (1972), 925-935.

[3] - Supersolutions, monotone iterations, and stability, J. Differential Equations, 21 (1976), 363-377.

[4] Chafee, N., Asymptotic behavior for solutions of a one-dimensional parabolic equation with homogeneous Neumann boundary conditions, J. Differential Equations, 18 (1975), 111-134,

[5] Friedman, A., Partial differential equations of parabolic type, Prentice-Hall, 1963.

[6] Fujita, H., On the nonlinear equations $\Delta u+e^{u}=0$ and $\partial v / \partial t=\Delta v+e^{v}$, Bull. Amer. Math. Soc., 75 (1969), 132-135.

[7] - On the asymptotic stability of solutions of the equation $v_{t}=\Delta v+e^{v}$, Proc. Intern. Conference on Functional Analysis and Related Topics, Tokyo 1969, Univ. of Tokyo Press 1970, 252-259.

[8] Itō, S., Fundamental solutions of parabolic differential equations and boundary value problems, Japan J. Math., 27 (1957), 55-102.

[9] - A remark on my paper "A boundary value problem of partial differential equations of parabolic type" in Duke Mathematical Journal, Proc. Japan Acad., 34 (1958), 463-465.

[10] Keller, H. B., Elliptic boundary value problems suggested by nonlinear diffusion processes, Arch. Rat. Mech. Anal., 35 (1969), 363-381. 
[11] Protter, M.H. and Weinberger, H.F., Maximum principles in differential equations, Prentice-Hall, 1967.

[12] Sattinger, D. H., Monotone methods in nonlinear elliptic and parabolic boundary value problems, Indiana Univ. Math. J., 21 (1972), 979-1000.

Note added after submission: After completing this work, the author got acquainted with the following paper: Casten, R. G., and Holland, C. J., Instability results for reac tion diffusion equations with Neumann boundary conditions, J. Differential Equations 27 (1978), 266-273.

It contains the same results as in our Theorem 5.1. Further it is shown that any nonconstant solution of (1.3) is unstable if $f(u)$ is convex or concave. But the problem of finding $f$ and $D$ with which there exists a nonconstant stable solution of (1.3) is still unsolved there, the answer to which can be found in our Theorem 6.2 and Corollary 6.3. 